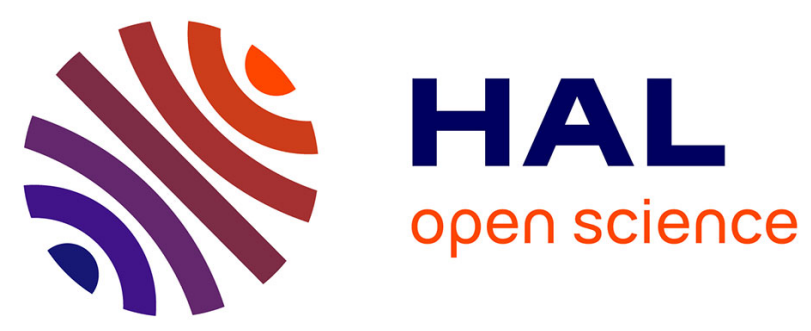

\title{
Les structures ornementales en acanthe dans les mosaïques de la villa de Séviac à Montréal (Gers)
}

Paulette Aragon-Launet, Catherine Balmelle

\section{To cite this version:}

Paulette Aragon-Launet, Catherine Balmelle. Les structures ornementales en acanthe dans les mosaïques de la villa de Séviac à Montréal (Gers). Gallia - Fouilles et monuments archéologiques en France métropolitaine, 1988, 45, pp.189-208. 10.3406/galia.1987.2886 . hal-01939605

\section{HAL Id: hal-01939605 \\ https://hal.science/hal-01939605}

Submitted on 14 Jan 2020

HAL is a multi-disciplinary open access archive for the deposit and dissemination of scientific research documents, whether they are published or not. The documents may come from teaching and research institutions in France or abroad, or from public or private research centers.
L'archive ouverte pluridisciplinaire HAL, est destinée au dépôt et à la diffusion de documents scientifiques de niveau recherche, publiés ou non, émanant des établissements d'enseignement et de recherche français ou étrangers, des laboratoires publics ou privés.

\section{(ㅇ)(1) $\$$}

Distributed under a Creative Commons Attribution - NonCommercial - NoDerivatives| 4.0 


\title{
LES STRUCTURES ORNEMENTALES EN ACANTHE DANS LES MOSAÏQUES DE LA VILLA DE SÉVIAC, PRÈS DE MONTRÉAL (GERS)
}

\author{
par Paulette ARAgON-LAUNET et Catherine BALMELLE
}

Dans le pays d'Armagnac, à douze kilomètres au n. d'Éauze, l'antique Elusa, capitale provinciale ${ }^{1}$, la villa de Séviac constitue un lieu privilégié, tant pour le spécialiste de la mosaïque que pour l'historien de l'Antiquité tardive.

Bon nombre de sites ruraux ont été révélés à différentes reprises sur le territoire de la commune de Montréal ${ }^{2}$, mais seule la villa de Séviac qui avait été signalée dès $1867^{3}$, a fait l'objet de campagnes de fouilles suivies et de travaux de mise en valeur et de restauration, ce depuis plus de vingt ans, sous la direction de l'un des auteurs de cet article, $\mathrm{M}^{\mathrm{mc}} \mathrm{P}$. Aragon-Launet ${ }^{4}$. Sise à $2 \mathrm{~km}$ au s.-o. de

1 Dans la Notitia Galliarum, Eauze figure comme capitale de la Novempopulanie, province qui regroupe les cités occupées par les peuples proprement aquitains, au s. de la Garonne.

2 Votamment au lieu-dit Glésia, situé à environ $3 \mathrm{~km}$ à l'e. de Montréal (sur cette villa fouillée à la fin du XIX" s., cf. en particulier, Abbé Cazauran, Bains gallo-romains à Montréaldu-Gers, Bulletin de la Socielé de Borda, XII, 1887, p. 249-268 et Abbé Brouirs, Villa gallo-romaine au Glésia près de Montréal-du-Gers, Revue de Gascogne. XXIX, 1888, p. 303314); d'autres sites antiques ont èté repérés, principalement à l'occasion de travaux agricoles: voir en dernier lieu, J. LAPART, Quelques découvertes archéologiques récentes dans le département du Gers, Bulletin de la Société archéologique, historique, lilléraire el scienlifique du Gers, 83, 1982, p. 131-139.

3 Par l'abbé Monnier, Curé de Labarrère, dans une lettre adressée à l'Empereur, en septembre 1867 (lettre conservée aux Archives des Monuments historiques, Paris, Dossier Gers, Affaires générales).

4 Des résumés des campagnes de fouilles ont été publiés par $M^{m *}$ P. Aragon-Launet dans le Bullelin de la Société archéologique, hislorique, litléraire et scienlifique du Gers (années 1959, 1962, 1971, 1974, 1980, 1983) ; par ailleurs, les principales découvertes ont été mentionnées dans les chroniques d'Informations archéologiques de Gallia (cf. en dernier lieu, Gallia, 41, 1983, p. 492-493). Les thermes sud dont la fouille a
Montréal, sur un plateau calcaire, de faible altitude, en pente sensible vers l'o., la villa aujourd'hui dégagée correspond à une grande maison de maître à péristyle, pourvue au s. d'un vaste complexe thermal implanté au fond d'une autre cour à portiques, intermédiaire entre le secteur du péristyle et les bains ${ }^{5}$. La mosaïque jouait un rôle important dans la décoration intérieure; outre les galeries et les couloirs, de nombreux espaces comportaient un sol en opus tessellatum ${ }^{6}$ : en particulier les salles de récep-

èté entièrement achevée viennent de faire l'objet d'une publication par R. Monturet et II. Rivicire (responsables de la fouille de ce secteur), Les thermes sud de la villa gallo-romaine de Séviac, suppl. 2 a Aquitania, Bordeaux, 1986; le mème ouvrage comporte une introduction due à $\mathrm{M}^{\mathrm{m}} \mathrm{P}$. AragonLaunet, qui présente un bref historique des fouilles de la villa (p. 14-15). Enfin, on trouvera plusieurs notices consacrées au site de Séviac dans le catalogue d'exposition, Premiers temps chréliens en Gaule méridionale, Antiquité tardive et IIaut Moyen

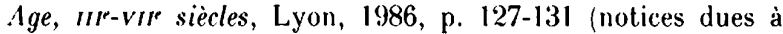
P.-A. Février, J. Lapart, et C. Balmelle).

5 Un plan schématique de l'ensemble de la villa, établi par R. Monturet (C.NRS, Institut de Recherche sur l'ArchitecLure Antique, Bureau du Sud-Ouest) d'après des relevés de II. Rivière et D. Matignon, est donné dans l'ouvrage de R. Monturet et II. Rivière (supra, note 4), pl. 3; on y trouvera également un relevé détaillé des thermes, pl. 4, ainsi qu'une série de plans illustrant les différents états reconnus. Un plan de la villa, toujours dû à R. Monturet, est publié dans C. Bat.Mri.t.F, Recueil général des mosaiques de la Gaule, IV, Province d'Aquitaine, 2. Pays gascons, Paris, 1987, X" suppl. à Gallia. Correspond à la figure 13.

6 L'ensemble des mosaïques de la villa est inventorié dans le fascicule 2 du tome IV du Recueil général des mosaiques de la Gaule (cité supra, note 5) sous les n" 285 à 310 : il s'agit de mosaiques de pavements, sauf dans le cas du n" 309 qui regroupe des éléments de mosaique pariétale, découverts pêlemêle dans un bassin des thermes. Sur les mosaiques des thermes, voir aussi l'étude par E. Monturet dans la publication de R. Monturet et II. Rivière (supra, note 4), p. 135-169. 
tion, les salles des thermes et la natatio du grand frigidarium.

Les données archéologiques jusqu'à ce jour réunies ne permettent pas de retracer l'histoire globale de la villa (qui a été fouillée surtout en surface) ni de dater avec précision les couches de l'Antiquité tardive. En ce qui concerne plus spécialement les mosaïques, il importe néanmoins de mettre en évidence plusieurs données issues de la fouille des thermes, des divers sondages et de l'examen attentif du support des pavements. Nous soulignerons, en premier lieu que pour l'une des mosaïques du grand frigidarium, nous disposons d'un terminus post quem vers $350-360^{7}$. D'autre part, il est clair que la décoration en mosaïque ne se rattache pas à un seul état de la villa : ainsi, dans les thermes, la mosaique parićtalc est obligatoircment antćricure au pavement du frigidarium ${ }^{8}$; par ailleurs, dans le secteur du péristyle, des sols mosaĩqués avaient été arrachés et remployés dans la construction, à en juger par les éléments de mosaique (tesselles isolées ou liées avec du mortier, fragment de radier) repérés dans le support de quelques tapis ${ }^{9}$; en outre, comme dans les thermes, des morceaux de mosaique avaient été utilisés comme matériau de comblement ${ }^{10}$. Pour ce qui est des pavements en place (grosso modo au même niveau de circulation), on peut se demander s'ils sont tous contemporains: les remaniements observés dans la plupart des pièces d'apparal, l'exislence de sols arrachés, les traits stylistiques de certains tapis inciteraient à établir une chronologie relative parmi ces pavements de l'Antiquité tardive ${ }^{11}$.

7 Dans la couche sous-jacente au support ont été recueillis une monnaie de Décence et des tessons de céramique dont les éléments les plus récents appartiennent au milieu du ive s: : C. Balmelle, Recueil mos. Gaule, IV, $2, n^{\circ} 307$; et pour plus de détails, R. Monturet, II. Rivitire, op. cit. (supra, note 4) p. 93-94 et p. 222 (ètude des monnaies par J.-P. Bost).

8 R. Monturet, II. Rivière, op. cit. (supra, note 1), p. $51-52,70$.

9 C. Batmelde, Recueil mos. Gaule, IV, 2 , nos 289,292 , 293. Un sondage récent, effectué en août 1986, a également mis en évidence la présence de tesselles dans le support d'une mosaïque de l'aile o. (n' 296 dans le Recueil).

10 Id., nos $294,310$.

11 Ainsi dans le Recueil, il a été suggéré que certaines mosaĩques à décor végétal pouvaient avoir été posées après celles du péristyle : nos $292,293,303 \mathrm{~A}$, peut-être aussi nos 289 , 295,296 . La datation proposée pour ces mosaïques (première moitié du ves.) reste naturellement hypothétique mais les recherches en cours sur l'ensemble du décor de la villa laissent. bien présumer qu'il faut placer postérieurement au Ive $\mathbf{s}$. la dernière phase d'embellissements de la demeure. D'autre part, en ce qui concerne plus précisément les thermes, on ajoutera que l'étude globale de l'architecture a conduit R. Monturet et
La décoration des pavements de la villa est à base d'éléments géométriques et végétaux qui appartiennent, à de rares exceptions près ${ }^{12}$, au répertoire des mosaistes aquitains de l'Antiquité tardive. Dans la présente étude, nous avons choisi d'attirer l'attention sur l'acanthe (nous allons revenir sur les problèmes d'identification), dans la mesure où le motif apparaît sur une série de tapis de Séviac, avec une forme spécifique, dans des structures variées dont l'étude conduit, pour certaines d'entre elles, à s'interroger sur les sources d'inspiration des mosaïstes de cette partie de la Gaule.

Les ornements végétaux, dans les créations décoratives, montrent souvent des formes simplifiées et schématiques, très éloignées des plantes réelles, plus spécialement à la fin de l'Antiquité : aussi leur identification à une espèce précise n'est-elle pas toujours aisée. Il convient donc de spécifier que dans le cas des mosaiqques de Séviac, le terme d'acanthe a été appliqué à toute représentation de tige portant des feuilles dentées, plus ou moins aiguës, à côtés curvilignes. Ajoutons cependant, que le terme a été aussi retenu pour certains rinceaux à gaines bractéales rencontrés sur d'autres pavements aquitains ${ }^{13}$. Ces diverses figurations végétales, dépourvues de naturalisme, restent, selon nous, liées à l'acanthe dont elles mettent en évidence deux principaux aspects, l'un plutôt foliaire, l'autre plutôt floral. Par ailleur's, compte tenu des nombreuses comparaisons établies avec une série de mosaiques, hors de l'Aquitaine, il nous a paru souhaitable de conserver la référence à cet ornement végétal, au demeurant si prisé dans l'art décoratif gréco-romain.

L'acanthe a été ainsi reconnue à Séviac sur six tapis: cinq d'entre eux sont concentrés dans le secteur du péristyle où ils décorent différents types d'espaces (galeries, exèdre, salles d'apparat); le dernier, enfin, se trouve dans la salle à double abside construite en bordure du portique 0 . de la cour intermédiaire. Toutes ces mosaïques se situent dans des espaces remaniés. Certainement postérieures au

11. Rivière à supposer que la mosaique de l'apodylerium avait été posée avant celle du frigidarium : cf. publication sur les thermes (supra, note 4), p. 59-60.

12 Par exemple la composition orthogonale d'octogones sécants et adjacents, traitée en filets triples bichromes, droits et dentelés: voir C. Balmelle, Recueil mos. Gaule, IV, 2, $n^{\circ} 301$, avec références sur ce mode de traitement.

13 Voir en particulier le rinceau illustré sur un pavement de la villa de Géou à Labastide-d'Armagnac (infra, p. 193 et fig. 2a). 
a

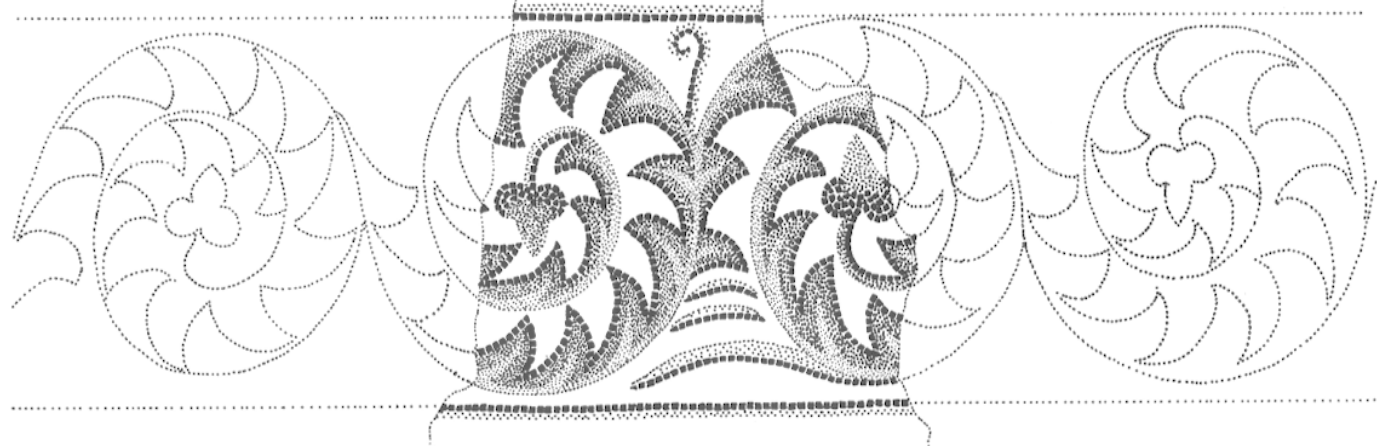

b

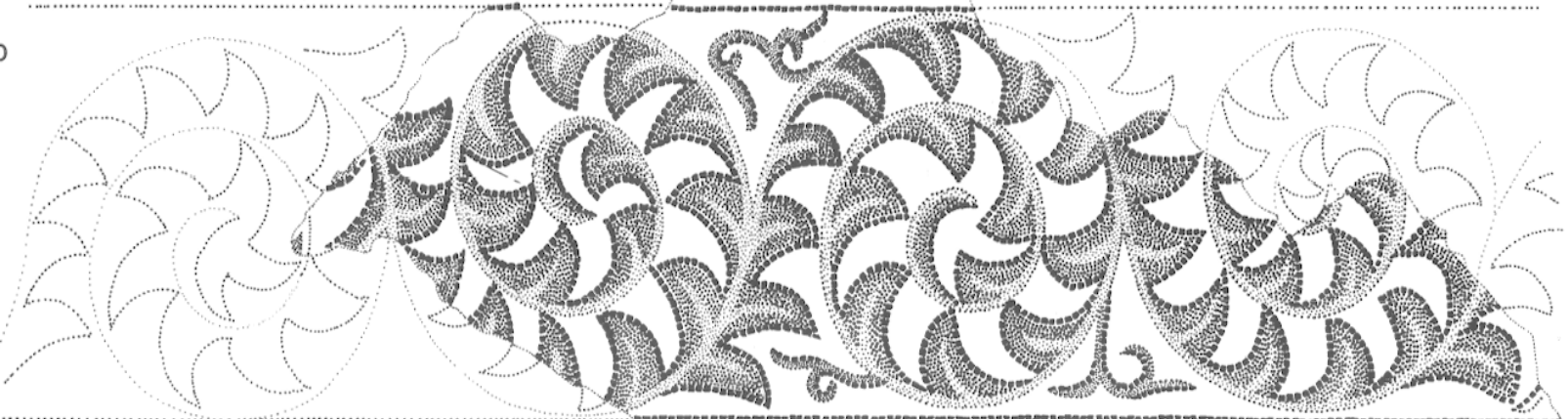

c

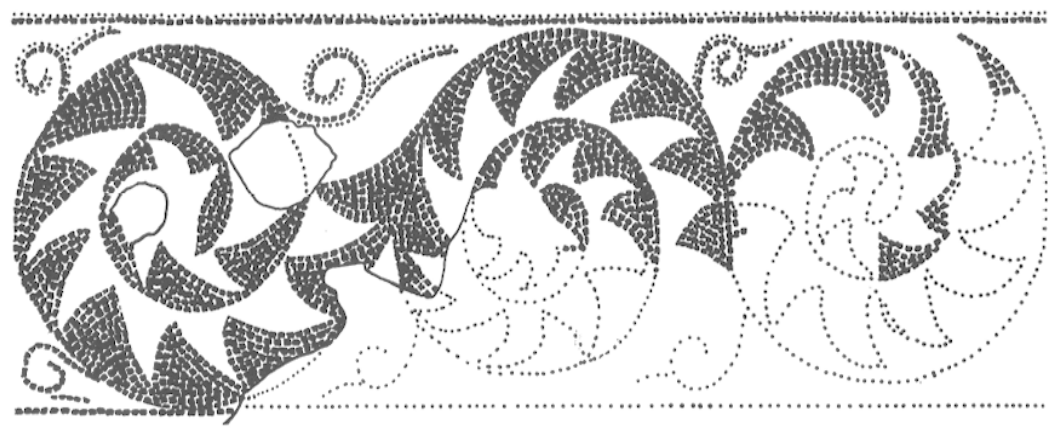

Fig. 1 - Rinceaux en feuilles d'acanthe à dents profondes et aiguës (type 1). Les dimensions indiquées entre parenthèses correspondent à la largeur de la bande blanche portant le rinceau. a, Séviac (largeur : $85 \mathrm{~cm}$ ); b, Taron (largeur : $55 \mathrm{~cm}$ ); c, Saint-Sever, pavement de l'abbatiale romane (largeur : $70 \mathrm{~cm}$ ).

milieu du IV $\mathrm{V}^{\mathrm{s}}$., elles pourraient appartenir à des phases distinctes de la décoration, ainsi qu'il a été suggéré plus haut ${ }^{14}$. L'acanthe témoigne à Séviac d'une grande souplesse d'utilisation : soit elle constitue une structure linéaire (rinceau), soit elle organise une composition de surface, sous forme de cercles en acanthe tangents ou bien sécants, soit encore elle intervient comme ornement associé à une pyramide végétale.

14 Voir supra, p. 190, plus spécialement note 11.

\section{L'ACANTHE EN COMPOSITION LINÉAIRE}

Des éléments d'un rinceau d'acanthe sont reconnaissables à l'extrémité o. d'une grande salle rectangulaire située au n.-o. du péristyle; le motif devait décorer un seuil occupant toute la largeur de la pièce $(6,40 \text { à } 6,80 \mathrm{~m})^{15}$. Très abîmé au moment de la découverte, aujourd'hui détruit, le rinceau (fig. 1a) montrait deux volutes d'environ $80 \mathrm{~cm}$ de diamètre,

15 C. Barmentre, Recueil mos. Gaule, IV, 2, n“ 295 (datation suggérée, seconde moitié du IV"s.). 
sur fond blanc, qui étaient dessinées par une tige (gris bleuté) prolongée intérieurement par des dents aiguës et resserrées (gris-vert, nervure centrale gris bleuté, cerne noir), ayant l'aspect d'épais triangles à côtés courbes; au centre de chaque volute, la tige se terminait par un pétiole portant une hedera rouge ou jaune, à base noire; une vrille et des filaments remplissaient les espaces triangulaires laissés libres.

Le rinceau d'acanthe a été souvent représenté dans les mosaïques tardives du Sud-Ouest de la Gaule, soit en motif de bordure extérieure du tapis, soit en motif de seuil comme ici ${ }^{16}$. Malgré des traits communs (schématisation extrême du végétal, développement en volutes, polychromie, fond blanc), les rinceaux montrent des variantes dans le rendu, les unes relatives au tracé même du rinceau, les autres concernant les remplissages. Autant que nous soyons en mesure d'apprécier le traitement de certaines mosaïques connues seulement par des dessins ${ }^{17}$, les rinceaux d'acanthe inventoriés en Aquitaine méridionale ${ }^{18}$ semblent se rattacher à trois principales catégories.

\section{Le rinceau en feuilles d'acanthe ì Dents PROFONDES ET AIGUËS}

Il peut être considéré comme représentatif d'un élément figuré de l'acanthe purement foliaire. La mosaïque de Séviac correspond à ce type qui regroupe le plus grand nombre d'exemples. Des rinceaux analogues ont été relevés à Saint-Mézard

16 Pour les exemples rencontrés en Aquitaine méridionale, voir infra, notes 19,20,21, 22, 25, 29,30. D'autres mosaiqques tardives du Sud-Ouest de la Gaule illustrent ce type de composition : ainsi à Moncrabeau-Bapteste dans le Lot-etGaronne, aquarelle due à $H$. Teulières, inédite (sur cette villa qui s'est révélée si riche en mosaïques, cf. G. LAFAYE, Inventaire des mosaiques de la Gaule, I, Narbonnaise et Aquitaine, Paris, 1909, nos 532 à 535); à Granéjouls dans le Tarn (G. LAFAYE, op. cit., $\mathrm{n}^{\circ \mathrm{s}} 384,386$, pl. correspondant au $n^{\circ} 386$ ); à Sana en Haute-Garonne (L. Joulin, Les établissements gallo-romains de la plaine de Martres-Tolosanes, Mémoires présenlés par divers savants à l'Académie des Inscriptions et Belles-Lettres, $1^{\text {re }}$ série, XI, 1901, p. 383, no 23 , mos. no 1); à Saint-Émilion, en Gironde (C. Balmelle, M. Gauthier, R. Monturet, Mosaiques de la villa du Palat à Saint-Émilion (Gironde), Gallia, 38, 1980, p. 69-75, fig. 6, 9 à 11, ve s.?).

17 Dans le cas des mosaïques de Cadeilhan-Saint-Clar et de Jurançon Pont d'Oly notamment, il est bien difficile de se rendre compte précisément du rendu : voir infra, notes 19 et 22 .

18 Il convient de spécifier qu'avec le fascicule $2 \mathrm{du}$ tome IV du Recueil général des mosaïques de la Gaule (supra, note 5) s'achève la publication du corpus des mosaiques de l'Aquitaine méridionale. dans le Gers ${ }^{19}$, à Pujo-le-Plan ${ }^{20}$, à Saint-Sever dans les Landes ${ }^{21}$ et à Taron dans les Pyrénées-Atlantiques (fig. 1b) ${ }^{22}$.

L'état fragmentaire de la plupart des rinceaux ne permet pas de savoir précisément comment se développait le motif. A Saint-Sever, les volutes paraissent se dérouler de façon continue. En revanche, à Taron, elles devaient être interrompues par un motif.

Le rinceau se compose de feuilles sans pétiole dont le limbe est réduit à des lobes superposés, pointus, en forme de dents. La nervure centrale de la feuille (l'axe du rinceau) présentée de face, en clair, se bifurque en deux volutes faisant apparaître une feuille de profil. Au centre de la volute, la nervure peut se terminer par une hedera (Séviac) ou par une fleur (Pujo-le-Plan). Une autre variantc rcncontrée à Saint-Mézard, à Taron et à Saint-Sever montre une feuille en languette comme élément terminal. D'ordinaire, la nervure centrale s'agrémente, dans les intervalles laissés libres, d'appendices en forme de vrilles. Dans certains rinceaux, l'espace résiduel est occupé par une feuille en languette et des filaments (Séviac), ou par un trois-feuilles (Saint-Mézard). Le rendu de l'acanthe est relativement homogène : nervure centrale tracée en clair ${ }^{23}$; dents aiguës avec nervure médiane, rendues à base de deux ou trois couleurs (dont gris, rouge, jaune, vert), cernées de noir; vrilles rouges et noires; les nervures des dents offrent souvent la même couleur que la nervure centrale de la feuille.

19 C. Balmelle, Recueil mos. Gaule, IV, 2, n* 440, description sommaire, sans illustration : la mosaĩque, inédite, devant faire l'objet d'une publication par J. Lapart. On trouvera cette mosaïque mentionnée dans Gallia, 44, 1986, p. 325-326, fig. 19. Les photographies qui nous ont été aimablement communiquées par J. Lapart permettent de constater que le rinceau est bien du même type que celui $\mathrm{dc}$ Séviac. Toujours dans le département du Gers, le pavement de Cadeilhan-Saint-Clar montre un rinceau d'acanthe qui pourrait, d'après les illustrations conservées, appartenir à cette série (Recueil mos. Gaule, IV, 2, n" 422).

20 C. Balmelle, H. Duday, B. Watier, L'établissement gallo-romain du quartier des Bignoulets à Pujo-le-Plan (Landes), Aquilania, 4, 1986, p. 205-221, fig. 8-9.

21 C. Basmelne, Recueil mos. Gaule, IV, 2, no 213.

22. Id., Recueil général des mosaïques de la Gaule, IV, 1 , Paris, 1980, no 110 . Le rinceau illustré sur une mosaïque de Jurançon Pont d'Oly (Pyrénées-Atlantiques) pourrait également se rattacher à ce type (Id., no 155 , pl. LXXXVII, aquarelle de Lecoeur).

23 La nervure est tracée en gris bleuté, en blanc ou en jaune; à Saint-Mézard, la nervure est partiellement doublée d'un filet noir. 

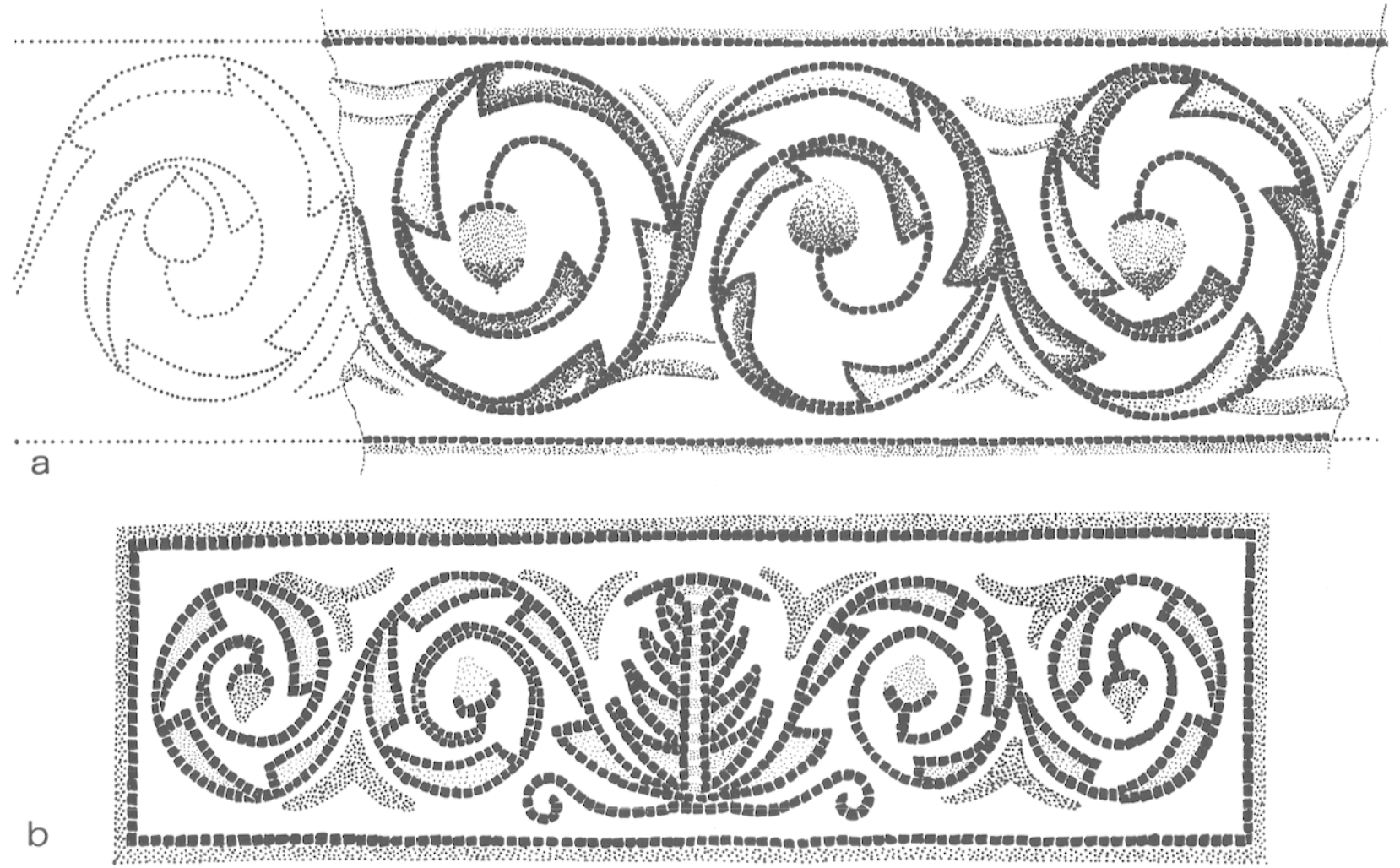

Fig. 2 - Rinceaux à gaines se terminant en pointe aiguë (type 2). a, Labastide d'Armagnac, Géou (largeur : $50 \mathrm{~cm}$ ); b, Moncrabeau, Bapteste.

\section{LE RINCEAU À GAINES SE TERMINANT EN POINTE AIGUË}

Malgré des similitudes de forme avec la série précédente, ce rinceau semble représenter une autre figuration de l'acanthe, essentiellement florale, les gaines (bractées, tiges florales) ${ }^{24}$. On le trouve à une seule reprise en Novempopulanie, dans la villa de Géou à Labastide-d'Armagnac dans les Landes (fig. $2 \mathrm{a})^{25}$; toutefois, un exemple pratiquement identique se rencontre sur le pavement d'une villa de la cité d'Agen, la villa de Moncrabeau-Bapteste située à environ $50 \mathrm{~km}$ à vol d'oiseau (fig. $2 \mathrm{~b})^{26}$. Dans les

24 On ne dissimulera pas cependant qu'il subsiste une ambiguité (gaine ou feuille?) due au fait que les éléments se terminent par une pointe aiguë qui rappelle les dents de l'acanthe.

25 C. Balmet.t., Recueil mos. (iaule, IV, 2, n" 245, avec référence à la récente publication de J.-P. Bost, P. Driond, E. et R. Monturet et II. Rivis̀re, La villa gallo-romaine de Géou à Labastide d'Armagnac (Landes), I, Les mosaïques, Bullelin de la Sociélé de Borda, 108. 1983, p. 403-441, en particulier p. 409-411, fig. 3.

26 Citée supra, note 16 . deux cas, le motif intervient comme décor de seuil : à Moncrabeau, le rinceau, connu seulement par une aquarelle, montre des volutes qui se développent symétriquement par rapport à un culot central; à Labastide-d'Armagnac, les trois volutes conservées n'autorisent pas à restituer avec certitude le développement.

Autour d'un fin pédoncule floral représenté dans sa partie terminale par une seule ligne de tesselles, s'emboîtent les unes au-dessus des autres des gaines imbriquées, presque de même taille, à l'extrémité en pointe aiguë. L'ensemble forme des volutes; au bout de l'axe pédonculaire, au centre de la volute, est figurée une sorte de bouton floral à base noire, de couleur rouge-rose ou jaune ${ }^{27}$. Les intervalles laissés libres entre les enroulements sont remplis de filaments ou de $\mathrm{V}$ rouges. L'axe pédonculaire et les gaines sont tracés en noir; les couleurs utilisées pour le remplissage des gaines varient d'un rinceau à l'autre.

27 Le motif pourrait peut-être aussi correspondre à une feuille de lierre stylisée. 

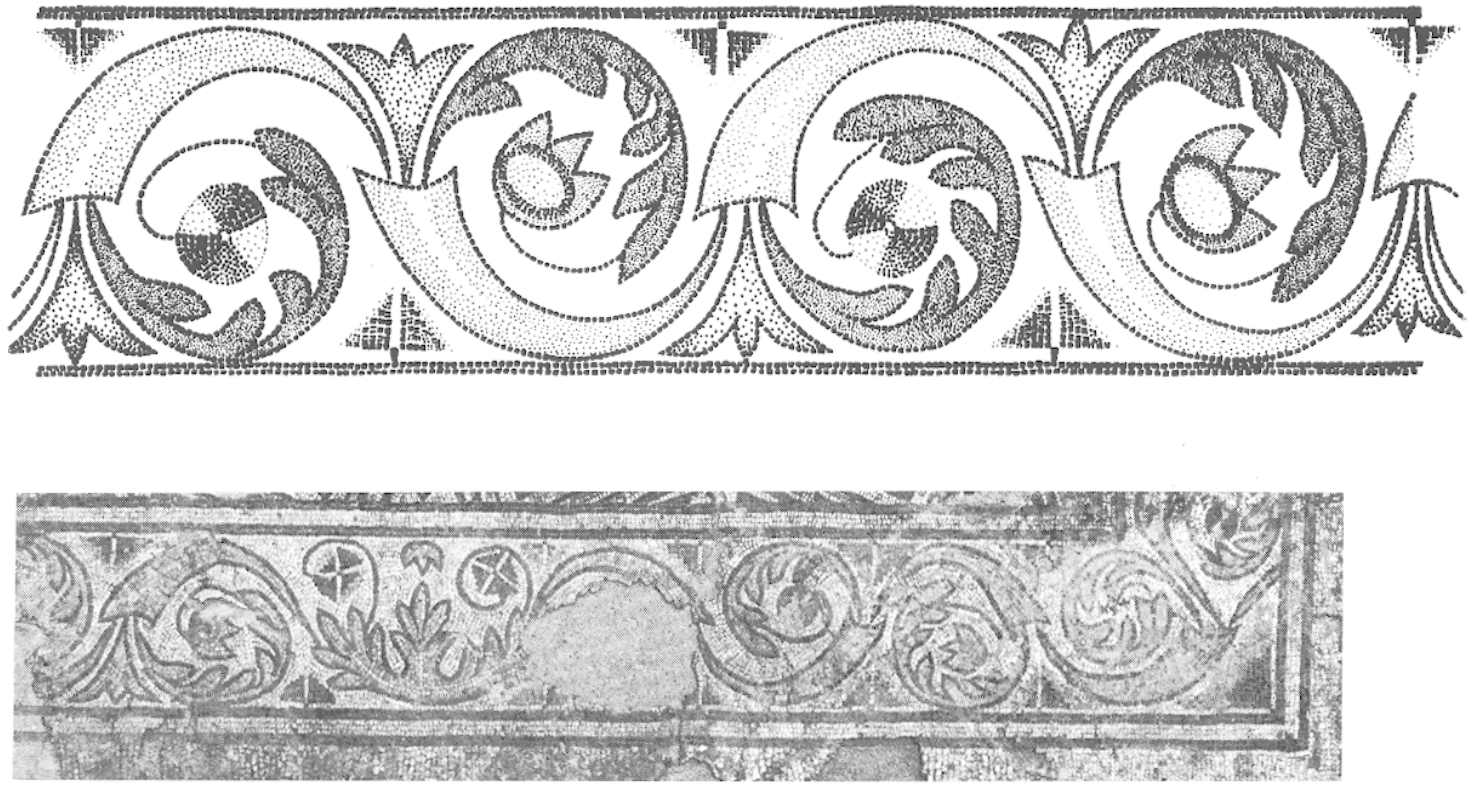

C

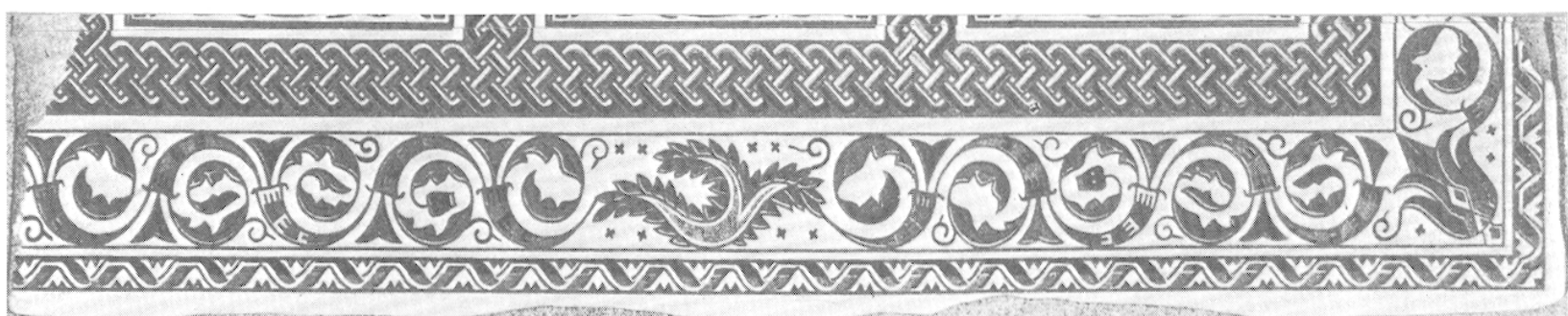

Fig. 3 - Rinceaux à gaines-cornucopiae et à feuilles d'acanthe (type 3). a, Hure (largeur : 40 à $50 \mathrm{~cm}$ ) dessin; b, Hure, mème rinceau, photographie avec vue du culot d'acanthe; c, Granéjouls, ensemble du rinceau délimitant l'un des côtés du tapis (aquarelle).

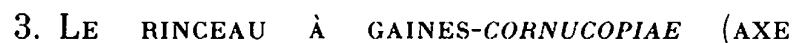
PRINCIPAL) ET À FEUILles D'ACANTHE (AXES SECONDAIRES)

Marqué par une très grande extension des gaines ${ }^{28}$, ce rinceau, tout en réunissant des éléments des deux séries précédentes, apparait comme un rinceau végétal de type mixte. Le même tracé des axes revient à Auch dans le Gers ${ }^{29}$ et à Hure en Gironde (fig. $3 a$ et $b)^{30}$. Toujours dans la région du Sud-Ouest, mais hors des limites de l'Aquitaine méridionale, il est attesté à Granéjouls dans le Tarn $(\text { fig. 3c })^{31}$.

28 Dans cette série, les gaines évoquent singulièrement des cornucopiae, bien qu'elles soient représentees sans embouchure, d'où notre expression gaines-cornucopiae pour les désigner.

29 C. Balmet.te, Recueil mos. Gaule, IV, 2, n”352.

30 Id., no 458.

31 Mentionné supra, note 16.
Motif de seuil (Auch) ou de bordure (Hure, Granéjouls), ce rinceau présente toujours un culot d'acanthe stylisé à partir duquel se développent symétriquement les ondulations. Les volutes sont nettement matérialisées mais elles n'offrent plus un tracé unitaire. L'axe principal en sinusoïde est dessiné par de larges gaines emboîtées ayant l'apparence de cornucopiae; une zone claire marque l'axe médian de la gaine (Hure, Auch), ce qui donne une impression de reflet. L'axe secondaire, en spirale, est constitué de feuilles d'acanthe, au limbe plus ou moins large et denté, qui jaillissent des embouchures des cornucopiae. La volute se termine par un pédoncule portant une fleur stylisée, de forme variable. Parfois, comme à Hure, l'élément terminal se compose d'un petit cercle divisé en quartiers, qui peut être également assimilé à un motif floral. Les écoinçons à l'extérieur des volutes sont garnis d'ornements végétaux : fleur de lotus, toujours dans l'axe de l'embouchure des cornucopiae (Hure, 
Granéjouls); demi-fleurette (Hure); feuilles en languettes (Auch); vrilles (Granéjouls).

Les différentes formes de rinceaux d'acanthe relevées dans les mosaiques tardives de l'Aquitaine méridionale ne semblent pas s'inscrire dans le cadre d'une évolution interne d'un type unique de rinceau, aux caractères bien définis. Il se pourrait, certes, que les variantes observées à l'intérieur des groupes 1 et 3 traduisent une évolution chronologique. Néanmoins, faute de disposer de datations précises pour l'ensemble de ces documents, nous devrons nous contenter de formuler certaines observations de simple vraisemblance.

Le rinceau composé de gaines se terminant en pointe aiguë (type 2) paraît correspondre à la forme la plus proche des "classiques" rinceaux à gaines figurés sur les mosaïques de l'époque impériale ${ }^{32}$. D'une diffusion limitée en Aquitaine méridionale, il peut être rattaché hypothétiquement à la production d'un atelier qui aurait participé, vers le milieu du IV's., à la décoration des villas de Labastided'Armagnac et de Moncrabeau ${ }^{33}$.

Le rinceau fait de gaines-cornucopiae emboîtees et de feuilles d'acanthe (type 3 ) retiendra à plusieurs titres notre attention. Il s'agit, semble-t-il, d'une structure assez rare et caractéristique de certains rinceaux de l'Antiquité tardive, marqués par une extension particulière des gaines qui tendent à se transformer en cornucopiae ${ }^{34}$. Les rinceaux offrant ce tracé spécifique des axes ont été principalement repérés sur des mosaïques de la Péninsule ibérique ${ }^{35}$

32 Voir par exemple, Kl. Pari.asca, Die römischen Mosaiken in Deutschland, Berlin, 1959 (Römischen-germanische Forschungen, XXIII), pl. 99-100. Ou encore J. Lancha, Recueil général des mosaïques de la Gaule, III, Province de Narbonnaise, 2, Vienne, Paris, 1981, n"367, pl. CV.

33 Cette hypothèse s'appuie également sur le fait qu'une mosaiqque de Moncrabeau (connue par une aquarelle, inédite) représente un décor strictement comparable à celui d'une mosaique de Labastide-d'Armagnac (Recueil mos. Gaule, IV, 2, n" 244); ces deux pavements sont aussi mis en parallèle par J.-P. Bost (voir article cité supra, note 25 ).

34 Cette tendance que l'on peut observer déjà aux IJ"IIr" s. notamment sur des mosaiques de la Gaule (cf. II. STrern, Recueil général des mosaiques de la Gaule, II, Province de Lyonnaise, 1, Lyon, Paris, 1967, n" 1, pl. IV-V), semble surtout caractéristique des rinceaux de l'Antiquité tardive : D. Lrvi, Antioch Mosaic Pavements, Princeton, 1947, p. 503-504; également G. Arkrstrom-Ilougren, The Calendar and Ilunting Mosaics of the Villa of the Falconer in Argos, A Study in Early Byzantine Iconograhy, Stockholm, 1974, p. 58-62.

35 En particulier à Mérida, A. Buanco FreiJeiro, Corpus de mosaicos romanos de España, I, Mosaicos romanos de Merida, Madrid, 1978, n" 43B, pl. 77, 102, 104 (seconde moitié du IV" s.). Également à Prado, F. Watrenbrirg, Los mosaicos de la villa de Prado, Boletin del Seminario de trle $y$ et de l'Afrique du Nord ${ }^{36}$, postérieures pour la plupart au milieu du IV ${ }^{e}$ s. Remarquons également que la même forme de rinceau apparaît au ProcheOrient sur l'un des tapis de l'église de la Nativité à Bethléem $^{37}$ et sur la mosaïque aux animaux des thermes de Serdjilla, datée de $473^{38}$.

L'ensemble de ces rinceaux montre naturellement des différences dans le rendu des éléments constitutifs (cornucopiae, feuilles d'acanthe), dans la couleur du fond, enfin dans le choix et le mode de traitement des motifs qui décorent l'intérieur des volutes et les espaces résiduels. Mais, on relèvera en même temps l'emploi d'un certain nombre d'ornements communs qui se retrouvent par ailleurs dans d'autres variantes tardives du rinceau d'acanthe. Ainsi de nombreuses mosaïques (Hure, Granéjouls ...) représentent une fleur de lotus jaillissant de l'embouchure des cornucopiae ${ }^{39}$.

Arqueologia, Valladolid, XXX, 1964, p. 121-127, fig. 1 et 3b (début du IV" s.?) et à Fraga, J. de C. Srrra Ráfoı.s, La villa Fortunatus de Fraga, Ampurias, V, 1943, p. 20-21, pl. X (fin du $1 v^{\circ} /$ début du V"s., cf. R. Pita Mrack, Mosaicos romanos tardios en las comarcas del Segre y Cinca, Boletin del Seminario ..., XXXV, 1969, p. 54-56).

36 Notamment à Althiburos, M. Ennaïfer, La cité d'Althiburos et l'édifice des Asclepieia, Tunis, 1976, p. 130, pl. CLII, fin du III/début du IV $\mathrm{s}$. (l'auteur pencherait à l'heure actuelle, suite à de nouvelles données archéologiques, pour une datation postérieure, dans la seconde moitié du Ive s.). Également à Sétif, P.-A. Février, Fouilles de Sétif. Les basiliques chrétiennes du quartier Nord-Ouest, Paris, 1965, fig. 139 (postérieurement au milieu du Ive s.) et à Djémila, M. Blanchard-Lemśe, Maisons à mosaiques du quartier central de Djémila (Cuicul), Aix-en-Provence, 1975, pl. XVI et LIb (sur les problèmes de datation, cf. P.-A. FÉvrier, Remarques sur les mosaïques de basse époque à Djémila (Algérie), Bulletin de la Socièté nationale des Antiquaires de France, 1965, p. 89-92).

37 L.-II. Vincent, Chronique. Bethléem. Le sanctuaire de la Nativité d'après les fouilles récentes, Revue Biblique, XLV, 1936, p. 543-574, pl. XI ; une datation dans la première moitié du ve $\mathrm{s}$. paraît très vraisemblable (cf. en dernier lieu, J. Balty, Les mosaiques de Syrie au ve siècle et leur répertoire, Byzantion, LIV, 1984, p. 448-449, en part. note 52).

38 D. Levi, op. cit. (supra, note 34), p. 504-505, fig. 184 (malgré la mauvaise qualité de la photographie, on peut lire la structure du rinceau).

39 Il convient de spécifier que le mème motif floral se trouve associé à d'autres formes de rinceaux d'acanthe sur les pavements tardifs de l'Afrique du Nord. On songera en particulier à un pavement des thermes du Pugiliste à Thina qui illustre un type de rinceau à cornucopiae bien attesté en Tunisie, d'une structure un peu différente de la nôtre, dans la mesure où l'axe principal est dessiné par une alternance de cornucopiae et de feuilles d'acanthe: cf. J. Thirion, Un ensemble thermal avec mosaiques à Thina ('Tunisie), Mélanges de l'École française de Rome, LXIX, 1957, p. 236 et suiv. pl. VI, fig. 1-4; d'autres exemples dans l'article de K. M. D. Dunbabin cité infra, note 41 . 
Enfin, de ces rinceaux d'acanthe à gainescornucopiae, il convient de rapprocher étroitement un groupe de rinceaux illustrés sur les mosaïques du Sud-Ouest de la Gaule. Remarquables par leur exubérance végétale, ils présentent tous une succession de cornucopiae bien caractéristiques, avec une embouchure évidée d'où s'échappent de lourds rameaux de feuilles en fuseau, souvent chargés de fruits $^{40}$.

La chronologie de l'ensemble de ces rinceaux aquitains faits de cornucopiae emboîtées reste encore aujourd'hui fort incertaine, faute de données archéologiques. Néanmoins, grâce à l'analyse stylistique, nous aurions tendance à placer cette production à une date relativement basse (fin $\mathrm{IV}^{\mathrm{e}} / \mathrm{V}^{\mathrm{e}} \mathrm{s}$.). Cela nous paraît plus spécialement évident pour la seconde série de rinceaux qui a perdu les traits essentiels du rinceau d'acanthe.

Le rinceau en feuilles d'acanthe (type 1) semble tout à fait spécifique du répertoire aquitain. Certes, les mosaïstes aquitains n'ont pas véritablement innové en développant cette structure de rinceau dépourvu de gaines et formé d'enroulements de tiges à dents aiguës d'une certaine épaisseur, représentées de profil. En effet, des caractéristiques analogues se retrouvent dans le rendu des rinceaux d'acanthe sur bon nombre de mosaïques tardives, notamment en Afrique du Nord ${ }^{41}$ et dans la Péninsule ibérique ${ }^{42}$. Néanmoins, l'examen approfondi de ces divers documents laisse nettement percevoir les traits bien particuliers du groupe du Sud-Ouest : par une série de détails, comme les lobes très épais avec une

40 C. Balmrlle, Recueil mos. Gaule, IV, 2, no 213 (Saint-Sever), avec références aux autres exemples aquitains; les feuillages, tout à fait atypiques, ne correspondent pas, selon nous, à de l'acanthe. Sur ces rinceaux, voir C. Balmelle, J. LAPART, La mosaïque à décor de pampres de Valence-surBaïse (Gers), article à paraître dans Aquitania, 5, 1987.

41 Par exemple à Carthage, dans la maison de la course de chars: R. II Anoune, Trois pavements de la maison de la course de chars à Carthage, Mélanges de l'École française de Rome, Antiquité, 81, 1969, p. 240-242, fig. 19 (au moins seconde moitié du $\mathrm{IV}^{\mathrm{e}} \mathrm{s}$.). On remarquera en outre la fréquence de ce type de rinceau sur les mosaïques de Carthage de la fin du Ive/début du ve s. : cf. K. M. D. Dunbabin, The Mosaics and Pavements, Excavations at Carthage conducted by the University of Michigan, I, Tunis, 1976, p. 24-29, pl. 7-9; également A. BEn Abed, Mosaíques provenant de l'aire du "Monument jouxtant la maison des auriges grecs", Centre d'Études et de Documentation archéologique de la conservalion de Carthage, 5 , 1983 , p. 23-25, fig. 3.

42 On songera plus particulièrement à une mosaïque funéraire de Barcelone: X. Barrai i Aitet, Les mosaíques romaines et médiévales de la Regio Laielana (Barcelone el ses environs), Barcelone, 1978, n" 24, pl. XXV,2 (trois derniers quarts du ve $s$. environ). nervure centrale de couleur claire, les mosaïstes aquitains ont su donner à ces rinceaux une forme spécifique qui met en évidence l'aspect foliaire de l'acanthe. En outre, la variante rencontrée à Taron, Saint-Sever et Saint-Mézard, avec une feuille en languette comme élément terminal de la volute, semble exceptionnelle hors d'Aquitaine ${ }^{43}$. En dernier lieu, il est intéressant de constater d'une part que certains sarcophages de l'École d'Aquitaine montrent des rinceaux d'acanthe offrant la même structure générale ${ }^{44}$ et d'autre part, que tous les rinceaux illustrés sur les mosaïques romanes de la région (fig. 1c) ${ }^{45}$ reprennent les caractères essentiels des rinceaux de ce groupe.

Enfin, il n'y a pas lieu de supposer une différence chronologique avec les rinceaux du second type. On pourrait seulement se demander si la variante mentionnée ci-dessus avec languette, qui se retrouve sur les mosaïques médiévales, n'est pas sensiblement postérieure à la variante comportant une hedera ou une fleur au centre de la volute, en apparence plus conforme à la représentation classique du rinceau d'acanthe.

\section{LA COMPOSITION DE CERCLES EN ACANTHE TANGENTS}

Dans deux tapis appartenant aux galeries $\mathbf{n}$. (fig. 4 et 5) et o. (fig. 6 et 7) du péristyle de Séviac, le décor du champ est constitué par une composition orthogonale de cercles en acanthe, tangents, déterminant des carrés concaves ${ }^{46}$. Aux points de contact des cercles, se trouve une sorte d'étui, en forme d'osselet évidé aux deux extrémités, au travers duquel passent les tiges d'acanthe.

Les cercles, de 1,15 à $1,20 \mathrm{~m}$ de diamètre, sont dessinés par des tiges prolongées intérieurement par

43 Une mosaïque de la villa tardive d'Ucero présente un rinceau d'acanthe avec des caractéristiques analogues mais le rendu et le style sont très différents : cf. J. M. BLAzQUFz, T. Orrego, Corpus de mosaicos de España, fasc. VI, Mosaicos romanos de Soria, Madrid, 1983, n 51, pl. 37 (début du ve s.).

44 Voir notamment B. Briesenick, Typologie und Chronologie der südwest-gallischen Sarkophage, Jahrbuch des römisch-germanischen Zentralmuseums, Mainz, 9, 1962, p. 76182, en particulier p. 116-120, pl. 27.1 (Toulouse, Musée des Augustins); également J.-B. Ward-Perkins, The Sculpture of Visigothic France, Archaeologia, 87, 1938, p. 124, pl. XXXVII, 5-6 (Toulouse, Musée des Augustins).

45 X. Barrat, i Atret, dans Recueil mos. Gaule, IV, 2, ${ }^{\text {os }} 3^{*}$ (Saint-Sever), 4* (Sorde-l'Abbaye).

46 C. Batmen.le, Recueil mos. Gaule, IV, 2, nos 285A, $286 \mathrm{C}$. 


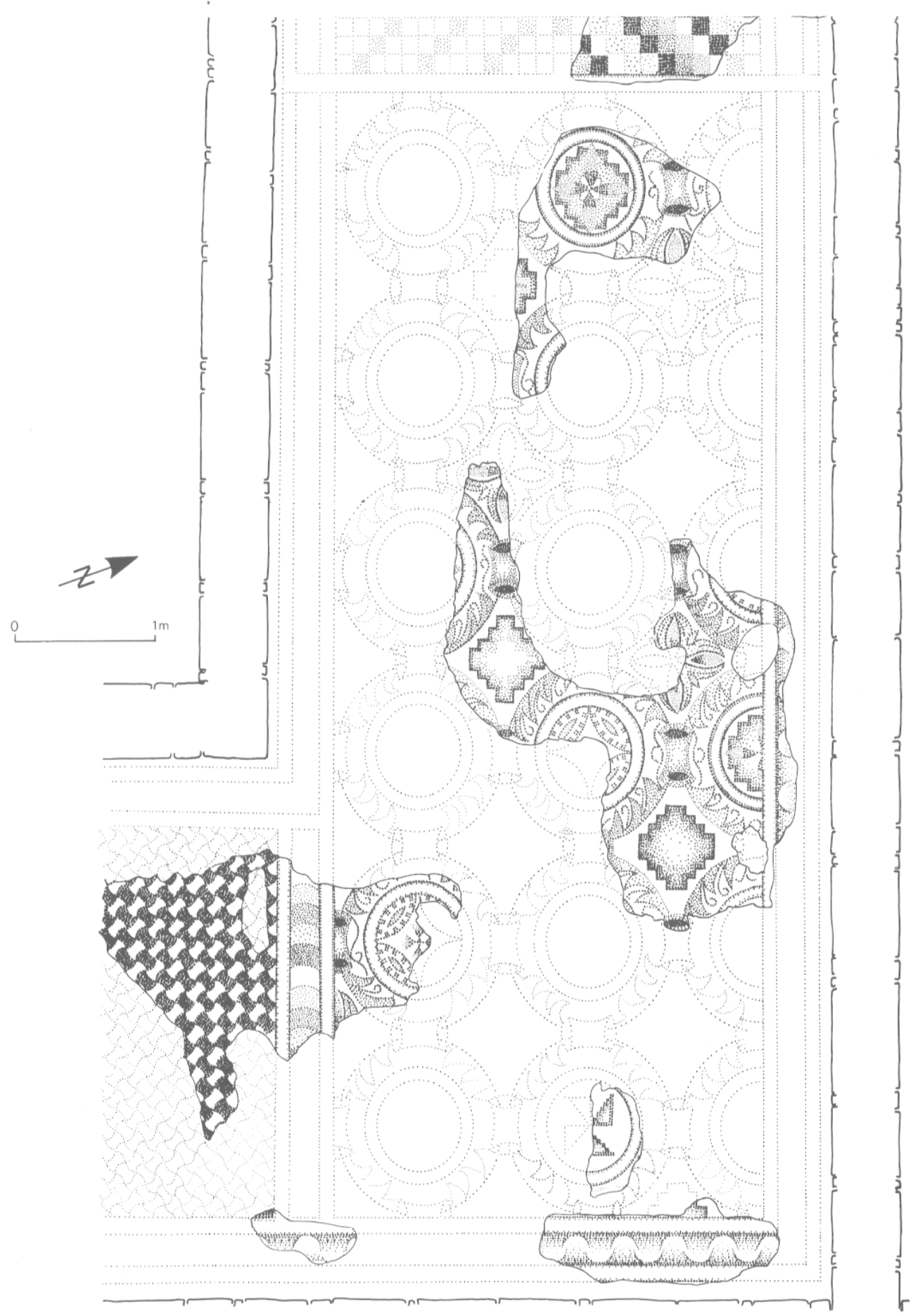

Fig. 4 - Séviac. Relevé de l'angle n.-e. du péristyle de la villa.

des dents aiguës et nervurées. Les couronnes ainsi formées présentent huit indentations dans la galerie o. et douze dans la galerie n. Sur les deux tapis, les feuilles, cernées de noir, sont gris-vert ou jaunes avec des nervures gris bleuté (marbre) ou rouges; des vrilles rouges se greffent sur chacune des couronnes.
L'exèdre située à l'e. du péristyle ${ }^{47}$ avait certainement reçu le même type de décor. Son pavement est malheureusement fort dégradé mais les éléments conservés ainsi que les empreintes des

$47 \quad I d ., \mathrm{n}^{\circ} 289$. 


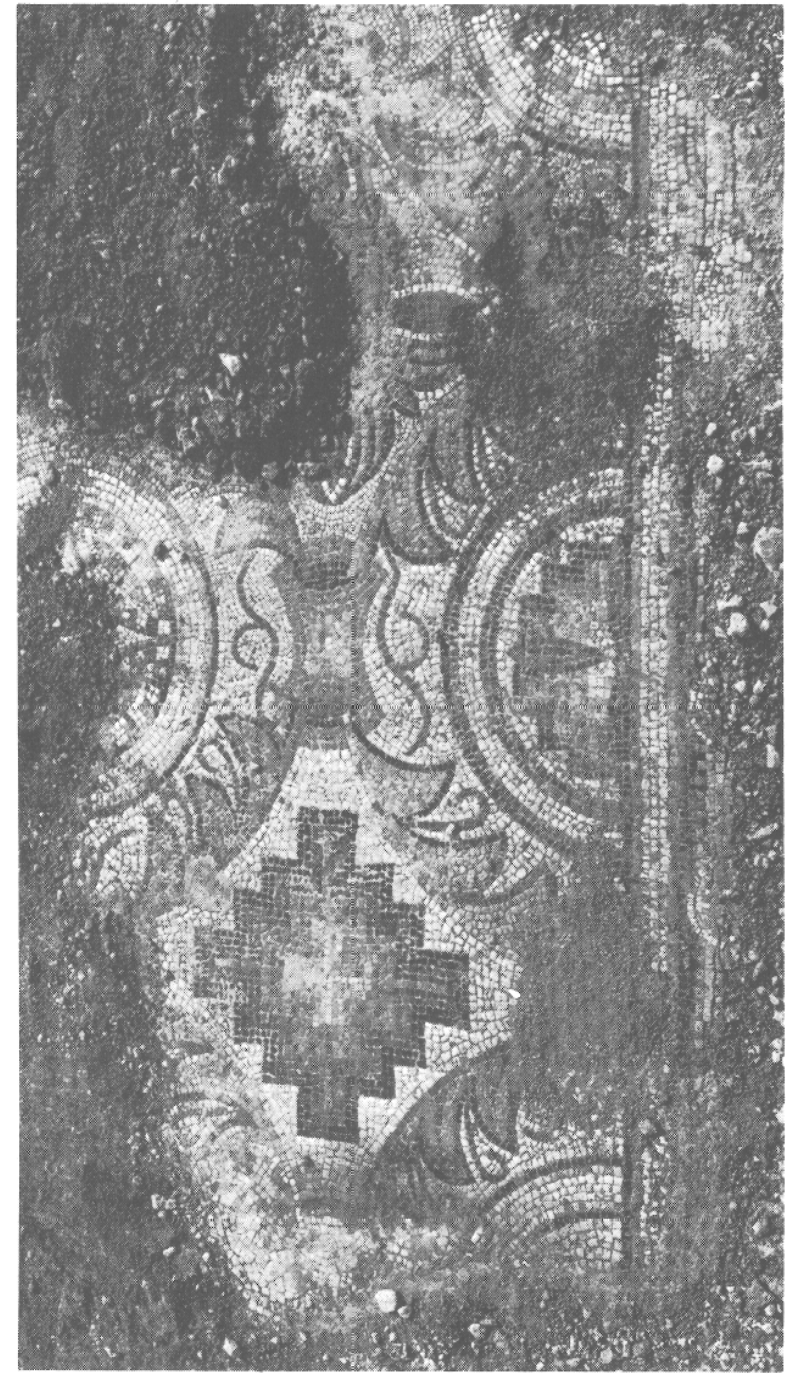

Fig. 5 - Séviac. Tapis à couronnes d'acanthe de la galerie $n$. du péristyle, in situ (1983).

tesselles restées lisibles dans le mortier nous ont permis de reconstituer une structure analogue, construite toutefois sur un module plus grand (cercles de $1,58 \mathrm{~m}$ de diamètre).

Les ornements de remplissage, dans les tapis des galeries 0 . et $n$. sont essentiellement géométriques (cercle de quatre fuseaux denticulés, carré à degrés, ailes de moulin, solides...) et végétaux (quatrefeuilles, fleurette, fleuron à quatre lotus séparés par quatre pétales en fuseau). Dans la galerie o., est représenté, en outre, à trois reprises, un vase à goulot $(\text { fig. } 6)^{48}$. Les rares remplissages qui subsistent dans

48 Sur les photographies prises in situ lors des fouilles de 1960-61, on distingue nettement un vase à panse arrondie, à deux anses et un vase à panse ovale, muni d'une seule anse; un autre vase était partiellement conservé.

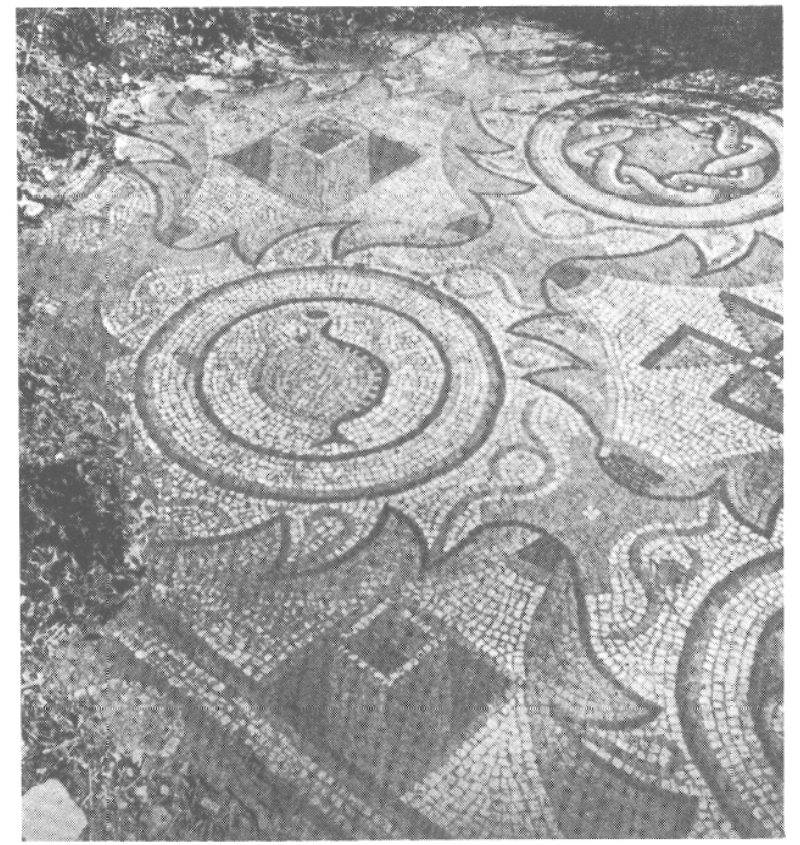

Fig. 6 - Séviac. Tapis à couronnes d'acanthe de la galerie o. du péristyle, in situ (1960).

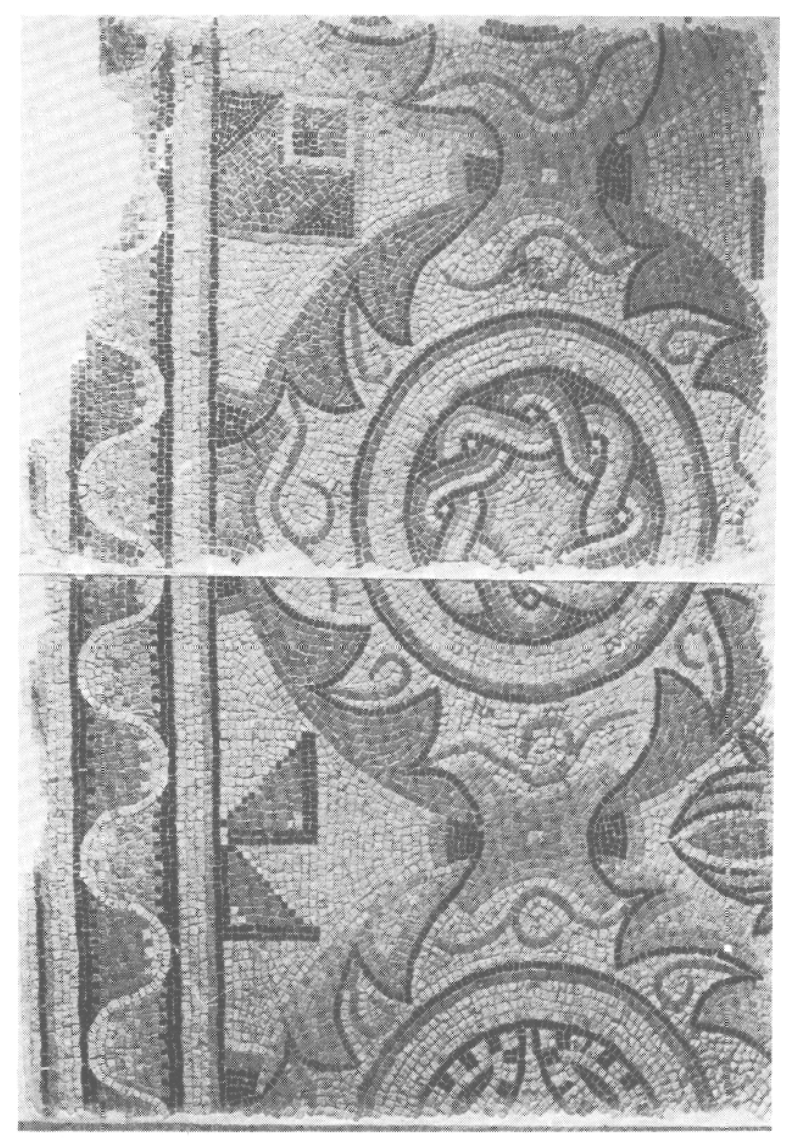

Fig. 7 - Séviac. Deux fragments du même tapis actuellement exposés au Musée de Montréal. 


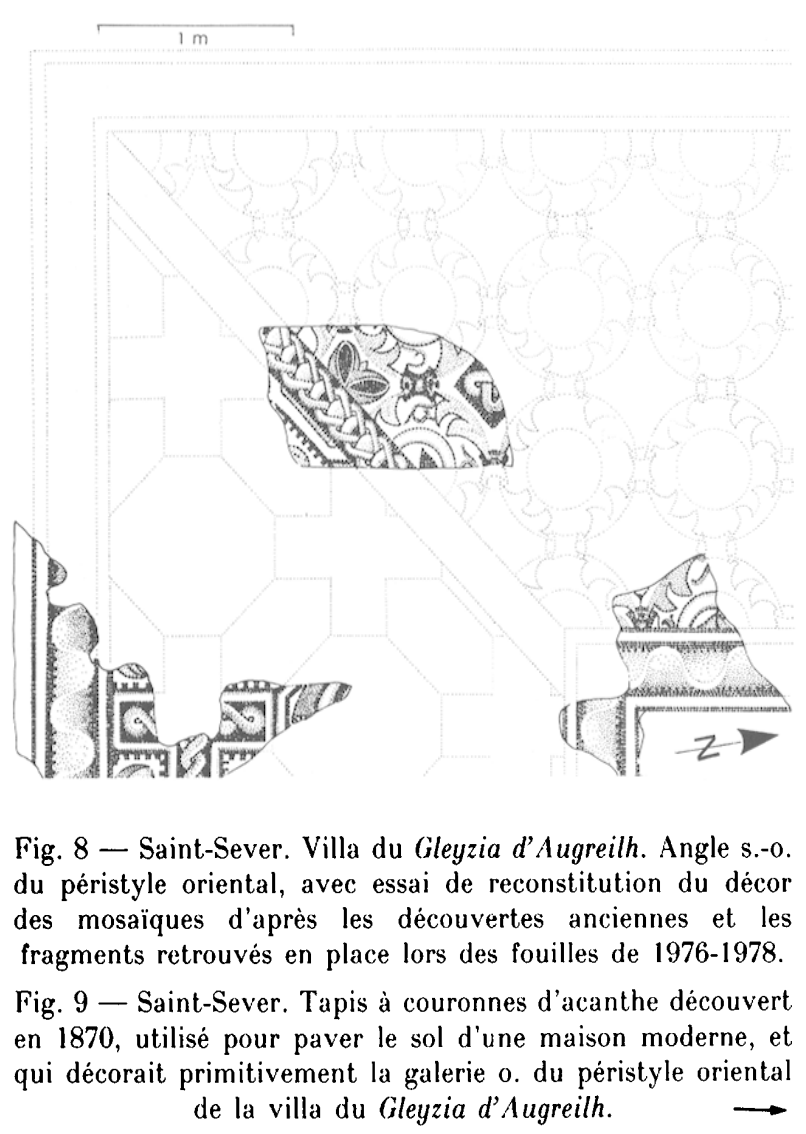

le tapis de l'exèdre sont purement géométriques : carré à fond noir, garni de quatre solides évidés (dans un carré concave); méandre fractionné et filet denticulé (dans les cercles).

Ces compositions de couronnes d'acanthe, illustrées également dans les villas voisines de SaintSever (Landes) (fig. 8 et 9 ) $^{49}$ et de Nérac (Lot-etGaronne $)^{50}$, avaient depuis longtemps retenu notre intérêt, en raison de leur caractère exceptionnel en Gaule et de leurs relations de ressemblance avec le répertoire africain ${ }^{51}$.

L'analyse comparative de l'ensemble des documents aquitains fait apparaitre un certain nombre de constantes dans le traitement de la composition : tiges à larges dents aiguës et nervurées, présence d'un étui évidé aux points de contact entre chaque couronne, remplissage à dominante géométrique et végétale. En dépit de ces similitudes, on relève des

49 C. Batmel.te, Recueil mos. Gaule, IV, 2, n" 208C.

$50 \mathrm{~J}$. Coupry, Informations archéologiques, Gallia, XXV, 1967, p. 363 , fig. 56 . La villa se situe dans le territoire de la cité des Nitiobriges.

51 C. Balmente, Les mosaiques de l'Aquilaine méridionale, thèse de III" cycle, Paris-Sorbonne, 1975, p. 362 et suiv.

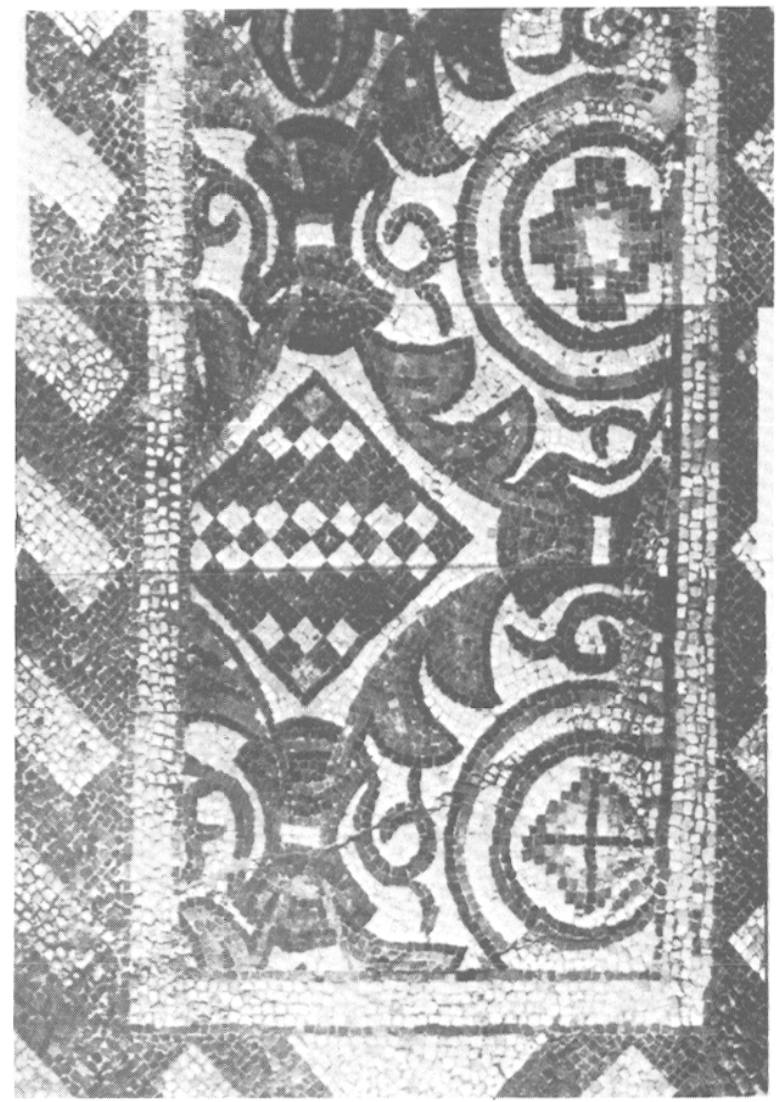

différences de style et de facture. Ainsi à SaintSever ${ }^{52}$, l'exécution est-elle nettement moins soignée qu'à Séviac. Le tapis donne une impression de surcharge, de lourdeur, due à la fois au petit module des couronnes à huit indentations (de 0,72 à $0,75 \mathrm{~m}$ de diamètre) et au rendu épais de certains ornements (par exemple les étuis cernés d'un filet double sépia, sur lesquels viennent se greffer de lourdes vrilles rouges et noires). A Séviac même, les deux mosaïques du péristyle montrent de légères variantes dans le mode d'exécution. Le tapis de la galerie o. se distingue par une composition très aérée, harmonieuse, avec des couronnes au feuillage moins dense et des cernes noirs, aux extrémités des ètuis, qui prolongent curieusement le contour des indentations. Quant à la mosaïque de Nérac, partiellement fouillée, dont le décor nous est connu seulement par une photographie, il est bien difficile de la situer par rapport aux autres exemples aquitains. Certains remplissages toutefois, comme la bordure en méan-

52 La mosaïque qui se trouve actuellement dans le vestibule d'une demeure de Saint-Sever, mal remontée, montre des restaurations modernes. Le fragment retrouvé en 1978 ainsi que les morceaux non restaurés, conservés dans la mème maison, ont permis toutefois d'apprécier le style du pavement. 

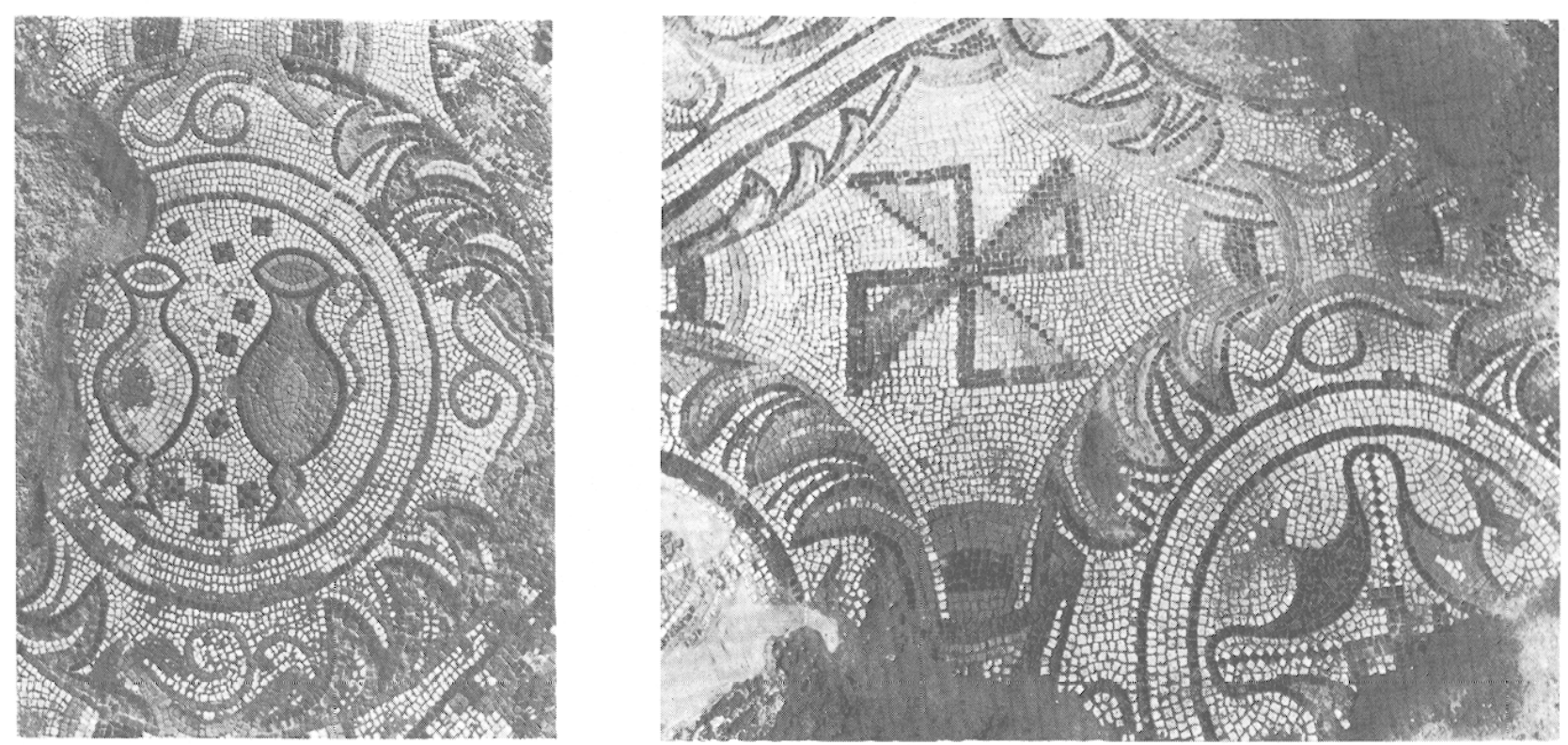

Fig. 10 - Loupian. Tapis à couronnes d'acanthe décorant l'une des absides de la salle triconque de la villa des Près-Bas, détails.

dre fractionné, suggéreraient un parallèle plus précis avec le tapis de l'exèdre de Séviac.

Dans le reste de la Gaule, ce mode de traitement végétalisé de la composition de cercles tangents a été repéré uniquement sur une mosaïque de la villa de Loupian (Hérault) (fig. 10 ${ }^{53}$. Les correspondances avec la série du Sud-Ouest, et plus spécialement de Séviac, sont si évidentes que Henri Lavagne avait déjà pu supposer que le tapis de Loupian avait été exécuté par une équipe itinérante de mosaïstes aquitains ${ }^{54}$.

La rareté de ces structures en couronnes d'acanthe dans les mosaiques des provinces gauloises, la nette concentration des occurrences dans le SudOuest indiquent de façon manifeste que nous sommes en présence d'un répertoire propre aux ateliers aquitains de l'Antiquité tardive. D'autre part, les recherches de documents de comparaison hors de Gaule montrent clairement que ces artisans se sont inspirés de mosaiques africaines à décor de couronnes de feuillages tangentes. Ces compositions végétalisées, sur lesquelles Gilbert Picard avait attiré

53 H. LaVAgne, Les nouvelles mosaĩques de la villa gallo-romaine de Loupian (Hérault), Revue Archéologique de Narbonnaise, XIV, 1981, p. 173-179, fig. 2 à 5.

54 Id., en particulier p. 179. H. Lavagne suppose qu'il s'agit plus précisément de l'équipe qui a travaillé dans la région de Montréal Séviac. Les différences de facture entre le tapis de Loupian et les tapis de Séviac nous inciteraient à penser que ce ne sont pas les mêmes artisans qui les ont réalisés. l'attention dès $1963^{55}$, se rencontrent avec une fréquence remarquable en Tunisie, du $\mathrm{II}^{\mathrm{e}} \mathrm{s}$. à la fin de l'Antiquité et témoignent de très nombreuses variantes selon la nature et le rendu des tiges feuillues qui matérialisent les couronnes ${ }^{56}$. La variante attestée en Aquitaine, avec des tiges à vrilles portant des feuilles unilatérales en forme de dents aiguës, que nous avons identifiées à de l'acanthe, trouve ses parallèles les plus proches dans des mosaïques tardives de Tunisie : en particulier à Thuburbo Majus (Maison des Protomés) (fig. 11) ${ }^{57}$, Utique (Maison de la Chasse) ${ }^{58}$, Bulla Regia ${ }^{59}$ et surtout Kélibia (Maison de Bir Ennahal) ${ }^{60}$. On relève, en outre, la présence de couronnes d'acanthe en dents

55 G. Picard, Un thème du style fleuri dans la mosaïque africaine, La mosaique gréco-romaine, Colloque international du CNRS, Paris, 1963, Paris, 1965, p. 125-136.

56 Les mosaiques de l'Afrique romaine à trames géométriques végétalisées ont fait l'objet de journées d'étude organisées à Tunis en septembre 1985, dans le cadre de la collaboration franco-tunisienne pour l'étude de la mosaïque de l'Afrique antique, établie entre le Centre de recherche sur la mosaique et le Comité tunisien pour l'étude de la mosaìque antique (GTEMA). Les Actes en seront publiés à l'École française de Rome sous forme d'un fascicule des Recherches franco-tunisiennes.

57 A. Ben ABed, A propos des mosaiques de la maison des Protomés à Thuburbo Majus, III Colloquio inlernazionale sul mosaico antico, Ravenna, 6-10 settembre 1980, Ravenne, 1984, p. 294-295, fig. 4 (terminus post quem dans la première moitié du Ive s.).

58 M. A. Alexander, M. Ennaïfer, J. Gretzinger, G. P. R. Metraux, D. Soren, M. Spiro, Corpus des mosaïques 


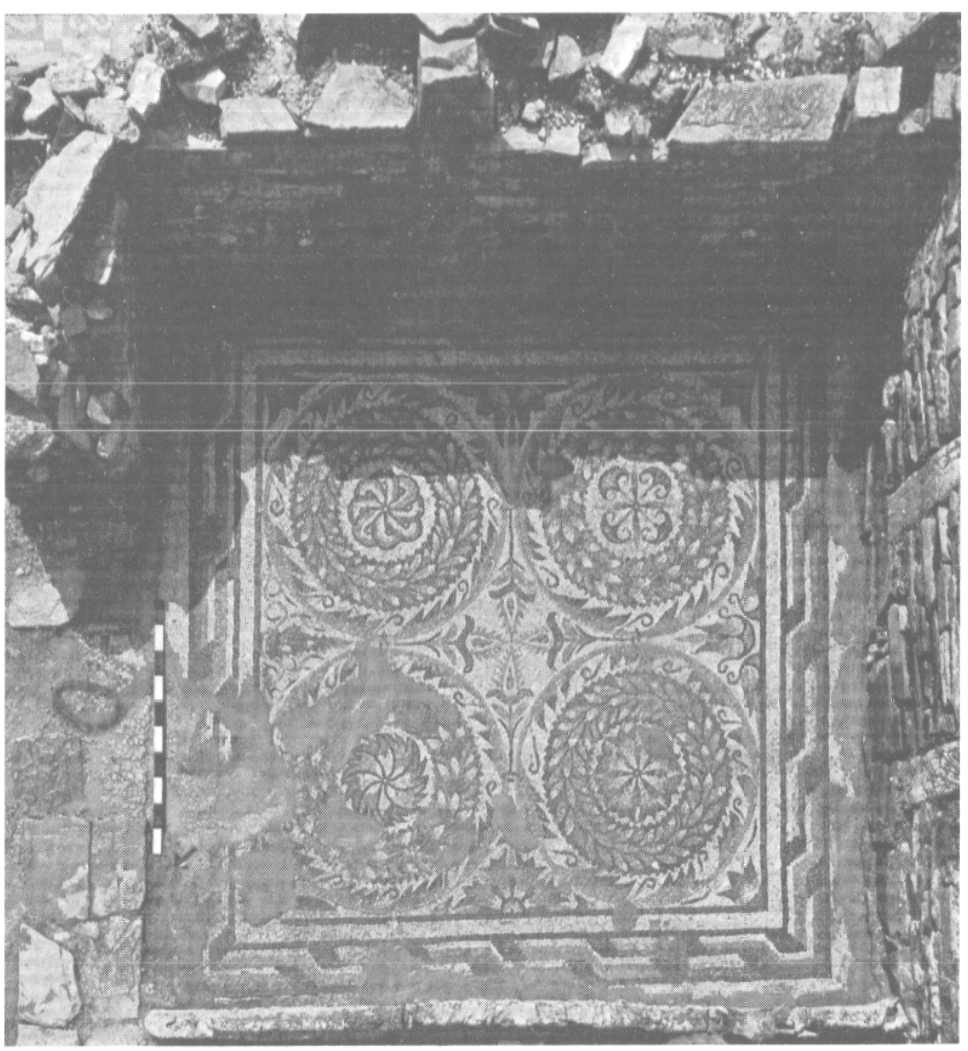

Fig. 11 - Thuburbo Majus, Maison des Protomés. Tapis à couronnes d'acanthe, in silu.

de scie, d'aspect très géométrique, sur les pavements d'époque byzantine, notamment dans l'église dite de Candidus à Haïdra (fig. 12) ${ }^{61}$.

Malgré ces rapprochements, il faut souligner qu'il n'existe pas de correspondance exacte entre les exemples africains et le groupe de la Gaule. Les tapis aquitains $^{62}$ se distinguent en premier lieu par l'emploi d'un ornement spécifique au point de contact des couronnes : il s'agit, comme nous l'avons

de Tunisie. I, fasc. 1, Utique, Insulae I-II-III, Tunis, 1973, $n^{\circ} 89$, pl. XXXIX (seconde moitié du IV $\mathrm{s}$.); on remarquera toutefois que les couronnes ne sont pas véritablement tangentes.

59 Mosaique se trouvant dans la maison n" 5 (inédite) 60 Cette mosaïque a été mentionnée par M. Ennaïfer lors du IV Colloque international pour l'Étude de la mosaique antique, Trèves 8-14 août 1984. Cette variante se retrouve sur une mosaïque de la villa Speciosa à Cagliari, d'aspect très africain, de la seconde moitié du ive s. : G. PIANu et alii, Villa Speciosa (Cagliari), Archeologia medievale, 9, 1982, p. 387-409, en part. p. 388-390, tav. III.3.

61 N. Duval, Y. Duval, L'église dite de Candidus à Haïdra (Tunisie) et l'inscription des Martyrs, Mélanges d'archéologie et d'histoire offerts à André Piganiol, II, Paris, 1966, p. 1153-1189, pl. III (époque byzantine).

62 Nous rattachons naturellement à ce groupe le tapis de Loupian. vu, d'une sorte d'étui évidé aux deux extrémités au travers duquel passent les tiges. Jamais rencontré dans la série d'Afrique du Nord ${ }^{63}$, cet ornement propre, semble-t-il, aux ateliers aquitains ${ }^{64}$, rappelle par son mode d'utilisation les éléments en vannerie que l'on voit associés à certaines compositions végétales, comme par exemple à Carthage, sur une mosaique décorée de sinusoïdes en tiges d'acanthe qui s'échappent de corbeilles ${ }^{65}$. Toutefois, le traitement de l'étui, sur les pavements de Gaule, ne correspond pas à un objet en vannerie. Il se pourrait que le mosaiste se soit inspiré de représentations de

63 Sur les mosaïques de Tunisie, on trouve au point de contact des couronnes d'acanthe en dents de scie, soit un lien, soit des vrilles, soit un fleuron longiforme orné de filaments. Dans certains cas, comme à Haïdra, aucun ornement particulier n'est représenté.

64 Il apparaît aussi associé à des représentations de rinceau végétal sur des mosaiqques (inédites) de la villa de Nérac (Lot-et-Garonne).

65 W. Bairem-Bren Osman, Calalogue des mosaiques de Carthage, Musée du Bardo, Musée des Thermes d'Antonin, Quartier de Dermech, Quartier de l'Odéon, Thèse de III ${ }^{\mathrm{C}}$ cycle, Université d'Aix-en-Provence, 1980, n" 97, p. 281-284. Cf. aussi S. Gozl.an, Deux mosaïques de Carthage au Kunsthistorisches Museum de Vienne, Mosaïque. Recueil d'hommages à Ilenri Stern, Paris, 1983, p. 183-184, pl. CXII, 1. 


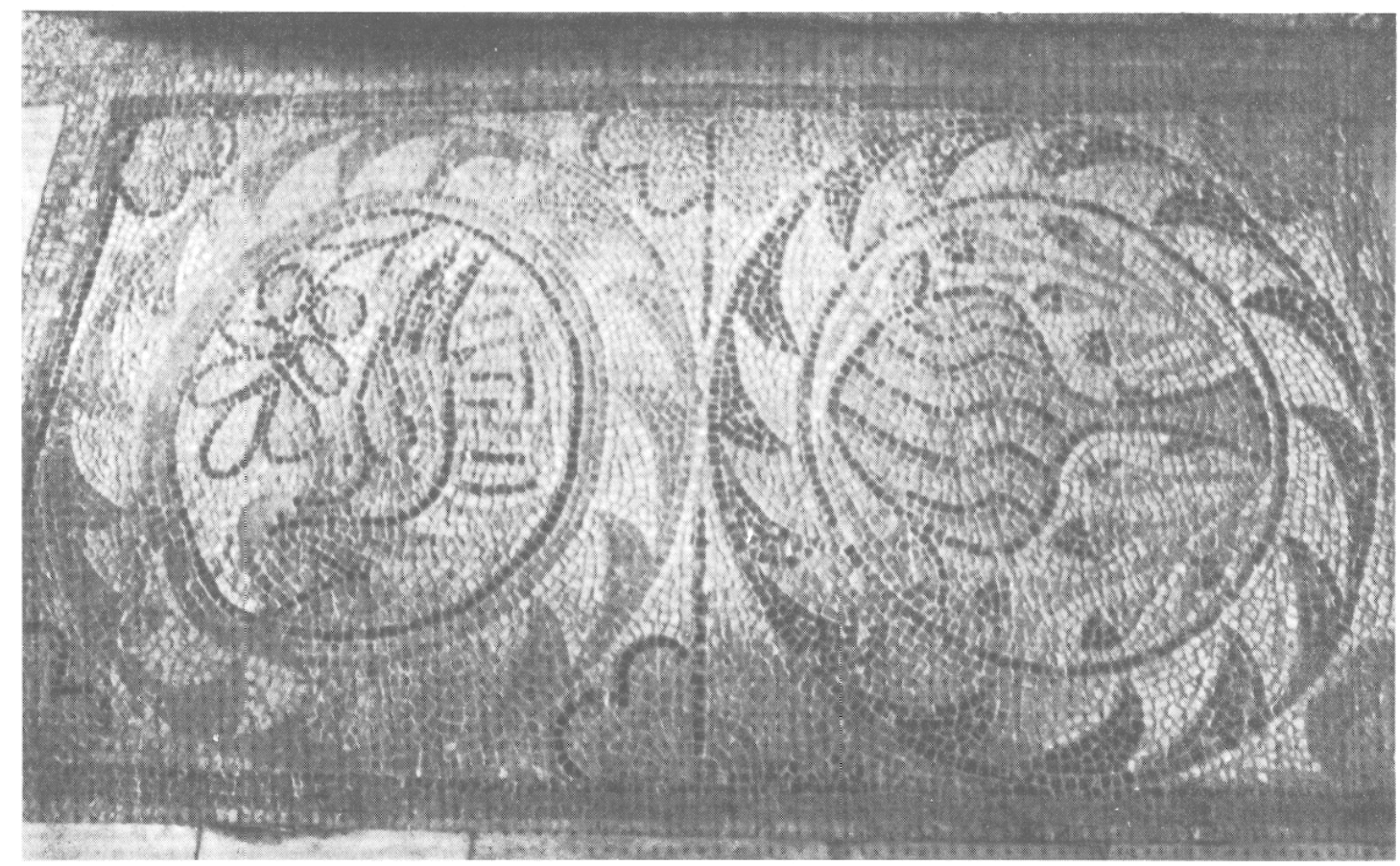

Fig. 12 - Haïra, église dite de Candidus. Panneau déposé et encastré dans le sol de la salle chrétienne du Musée du Bardo.

vases, l'osselet évidé, encadré de vrilles, suggérant la forme d'un cratère avec des anses à volutes ${ }^{66}$.

Une autre différence concerne le rendu des tiges d'acanthe. Dans le groupe aquitain, très homogène, les dents à sommet aigu sont très épaisses, cernées de noir, avec une nervure centrale très marquée. Les couronnes d'acanthe en dents de scie repérées en Nfrique, autant que nous puissions en apprécier le traitement, offrent un aspect sensiblement différent: les dents paraissent moins épaisses et ne présentent pas les caractéristiques relevées ci-dessus.

Enfin dans les ornements de remplissage, si quelques motifs sont communs aux deux séries (fleuron, fleurette, carré à degrés, ailes de moulin), d'autres au contraire, semblent plus spécifiques de l'une ou l'autre série : ainsi la couronne de laurier pour les exemples africains, les solides et les cercles de quatre fuseaux denticulés pour les exemples aquitains. Mais notre analyse s'applique à un petit nombre de documents et il va de soi que ces dernières remarques ont un aspect incomplet et provisoire.

66 On pensera plus particulièrement au cratère représenté sur l'un des pavements de la villa de Nérac (Gallia, 29, 1971, p. 355, fig. 36).
En définitive, les pavements à compositions de couronnes d'acanthe reconnus en Gaule apparaissent comme une libre transcription par les mosaïstes aquitains de structures végétalisées spécifiques du répertoire des ateliers de l'Afrique du Nord et plus particulièrement de Proconsulaire. La forme de couronne en dents de scie s'inscrit incontestablement dans l'évolution tardive de ces décors, encore attestès au $\mathrm{VI}^{*} \mathrm{~s}$, mais sous un aspect nettement géométrisé. Le groupe de la Gaule ${ }^{67}$, bien qu'homogène, montre des différences de style et de facture qui laissent penser que ce répertoire n'est pas le reflet d'un seul et unique atelier.

\section{LA COMPOSITION DE CERCLES EN ACANTHE SEGANTS}

A l'o. de la cour intermédiaire de la villa de Séviac, la salle à double abside (en grande partie recouverte par une construction moderne) montre dans l'abside n. un pavement fragmentaire dont le

67 Toujours dans le Sud-Ouest, on remarquera la représentation de couronnes d'acanthe, mais cette fois comme motif de remplissage, sur des pavements de Sarbazan et d'Auch (C. Balmelle, Recueil mos. Gaule, IV, 2, nos 251, 357). 

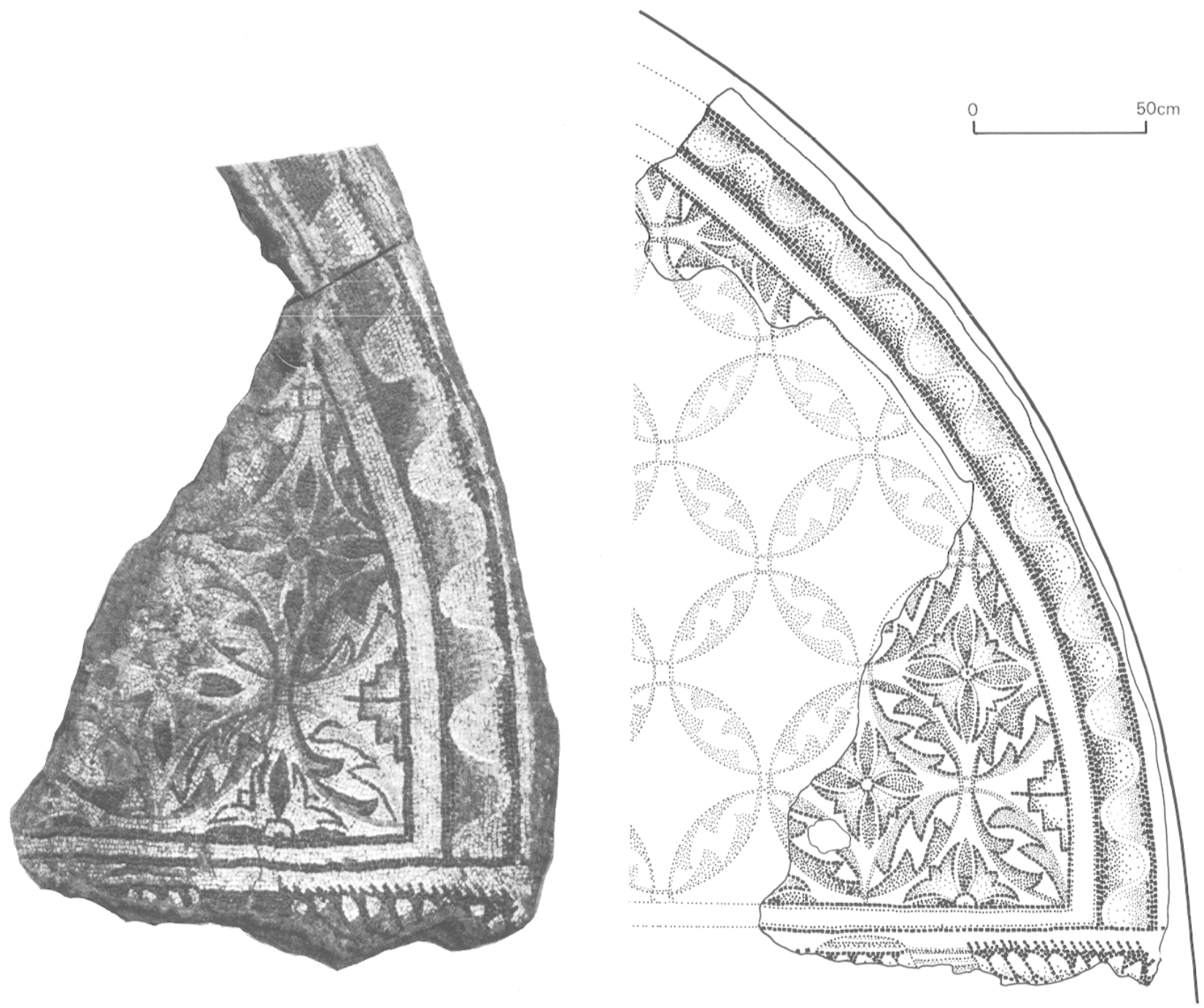

Fig. 13 - Séviac. Tapis à décor de cercles en acanthe sécants qui appartient à l'abside n. d'une salle à double abside. Photographie du principal fragment et relevé de l'ensemble (le nord est en haut).

décor est toutefois facilement identifiable ${ }^{68}$. Il s'agit d'une composition de cercles en acanthe sécants, faisant apparaître des quatre-feuilles. Les cercles $(56$ à $58 \mathrm{~cm}$ de diamètre) ne sont pas véritablement contigus, ce qui détermine un petit carré concave (au centre des quatre-feuilles) et des octogones irréguliers curvilignes (fig. 13). Les cercles, tracés en jaune ou en gris-beige, se prolongent intérieurement par huit indentations, au contour noir, colorées en jaune et rouge ou en gris-beige et gris bleuté (marbre). Chaque fuseau des quatre-feuilles est ainsi formé de deux tiges à deux indentations opposées et décalées. La polychromie est identique à celle des cercles en acanthe tangents. Les indentations ont une forme sensiblement différente, plus mince et étirée, ce qui peut d'ailleurs s'expliquer par le petit module des

68 C. Barmetr.e, Recueil mos. Gaule, IV, 2, no $303 \mathrm{~A}$. cercles. D'après les éléments conservés dans les octogones irréguliers, le remplissage se compose principalement de fleurons polychromes à quatre lotus alternant avec quatre feuilles en fuseau, autour d'un gros cœur circulaire. Sur la ligne de chute, figure également un carré à degrés, tronqué.

Ce type de décor semble, jusqu'à présent, d'un emploi limité dans les mosaïques de l'Aquitaine méridionale. Le principal document de comparaison est fourni par une mosaïque, malheureusement détruite, de la villa de Cadeilhan-Saint-Clar (Gers), située non loin de Séviac ${ }^{69}$. Une autre mosaïque récemment découverte à côté d'Auch ${ }^{70}$ offrait peutêtre une composition végétalisée analogue. Néanmoins, les petits fragments que nous avons pu voir

69 Id., $\mathrm{n}^{\circ} 422$.

$70 \quad I d ., \mathrm{n}^{\circ} 377$. 

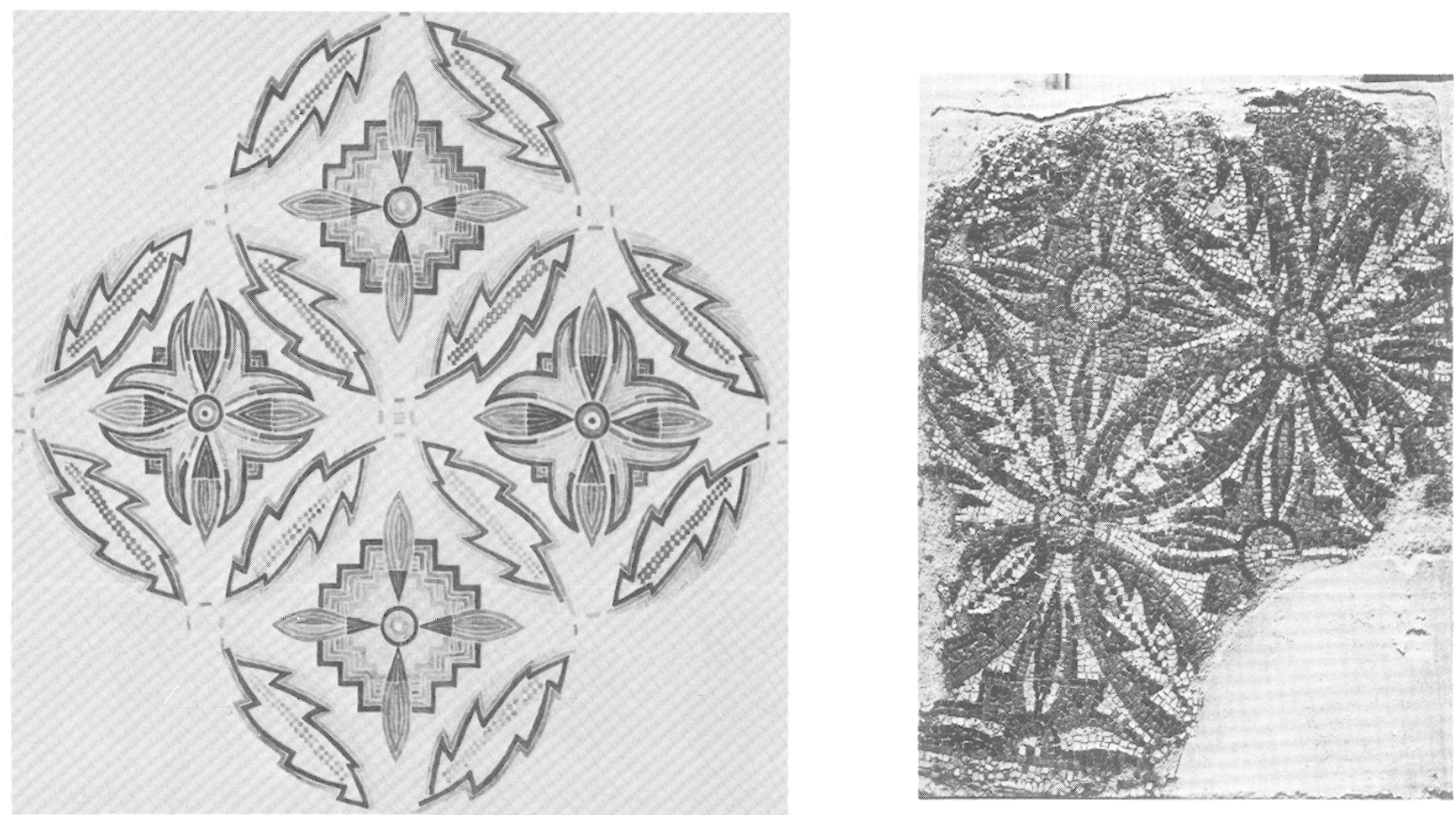

A gauche. Fig. 14 - Cadeilhan Saint-Clar. Aquarelle représentant un détail du tapis avec des cercles en acanthe sécants qui décorait l'abside d'une salle d'apparat de la villa.

A droile. Fig. 15 - Mosaiqque du baptistère souterrain de Carthage-Sayda, avec décor de cercles en acanthe sécants.

ne permettent pas de restituer le canevas avec certitude.

A Cadeilhan-Saint-Clar, le tapis de cercles sécants en acanthe (fig. 14), qui se trouve dans l'abside en fer à cheval d'une salle réception, se rapproche du tapis de Séviac par une série de détails : petit module des figures, agencement des cercles non contigus, forme des indentations, fleuron à l'intérieur des octogones irréguliers. Des différences s'observent toutefois dans la polychromie (cercles tracés en blanc) et surtout dans l'ornementation des fuseaux, garnis d'un filet axial dessiné par un filet triple dentelé.

Cette variante végétalisée de la composition orthogonale de cercles sécants n'a jusqu'à présent jamais été signalée en Gaule el mérite qu'on s'y arrête dans la mesure où elle revient, avec des caractéristiques analogues dans le traitement du végétal (feuilles en forme de dents de scie), sur une série de mosaïques tardives appartenant à certaines régions de la Méditerranée occidentale (Afrique du Nord, Sardaigne, Sicile, Campanie, Italie du Nord et Moyen Adriatique). Plusieurs spécialistes travaillant sur l'Afrique ${ }^{71}$, l'Italie du Nord ${ }^{72}$ et la Sicile ${ }^{73}$ ont

71 V. Duval, P.-A. Février, Le décor des monuments chrétiens d'Afrique (Algérie, 'Tunisie), Actas del VIII Congreso internacional de Arqueologia cristiana, Barcelona, 1969, RomeBarcelone, 1972, p. 20, fig. 13. attiré, à différentes reprises, l'attention sur ce type de décor et nous nous bornerons ici à formuler quelques remarques d'ordre général ${ }^{74}$.

Si l'origine africaine des compositions végétalisées de cercles en acanthe sécants semble devoir être retenue, en revanche, il est encore bien difficile,

72 R. Farioli, Pavimenti musivi di Ravenna paleocrisliana, Ravenna, 1975, en particulier p. 191-198, fig. 10, p. 27, fig. 100, p. 194, fig. 101, p. 196. En dernier lieu, S. Lusuard Sifna, La pavimentazione musiva della Cattedrale di S. Maria a Luni, Scrilti in ricordo di Graziella Massari Gaballo e di Umberto Tocchetti Pollini, Milano, 1986, p. 303-322, fig. 6-7, 15.

73 R.J.A. Wilson, Roman Mosaics in Sicily: The African Connection, American Journal of Archaeology, 86, 1982, p. $422-428$, fig. 21 à 24 . Il importe de ne pas retenir le second exemple de Cherchel (cité p. 424 et note 90), dans la mesure où l'article de $\mathrm{P}$. Mijovic évoqué en référence donne en réalité une photographie du pavement de Pomposa.

74 On trouvera une liste des exemples facile à consulter dans l'article récent de S. Lusuardi Siena (supra, note 72), p. 316-318; pour la bibliographie concernant Pesaro, on citera désormais l'étude de R. Farioli Campanati, Il Duomo di Pesaro tra Tarda Antichità e Medio Evo, Studia Oliveriana, IIIII, 1982-1983, p. 131-168, fig. 1. D'autre part, quelques exemples doivent ètre ajoutés à cette liste : Villaspeciosa en Sardaigne (infra, note 75); Hippone (infra, note 75) et Le Kef (inédit), en Afrique du Nord; enfin Zadar en Yougoslavie (M. Suic, Zadar u starom vijeku, Zadar, 1981, p. 331-334, pl. XXXV et pl. couleur entre p. 320 et 321 ). Je remercie très vivement . Noël Duval qui nous a signalé ces deux derniers exemples et qui a eu la grande gentillesse de relire ce texte. 
faute de chronologies serrées, de suivre le développement de la variante tardive marquée par des tiges à dents aiguës, par un filet axial (le plus souvent dentelé) à l'intérieur des fuseaux et par un petit cercle recouvrant aux points de tangence. Pour autant que la datation de la mosaïque de Villaspecio$\mathrm{Sa}^{75}$ soit assurée, cette variante apparaîtrait dès le IV $^{*}$ s. dans une ambiance africaine ${ }^{76}$. Repérée au V" $\mathrm{s}$. dans quelques édifices chrétiens de l'Adriatique et peut-être de Carthage ${ }^{7}$, elle a été principalement illustrée sur les pavements des églises du vir s., dans l'Adriatique, en Sicile et en Tunisie ${ }^{78}$. Les remplissages, constitués à l'origine de motifs exclusivement végétaux et géométriques, comportent souvent à l'époque byzantine des sujets animaliers.

Si nous confrontons les exemples du Sud-Ouest de la Gaule avec l'ensemble des documents illustrant cette variante telle qu'elle a èté définie plus haut, plusieurs remarques s'imposent.

75 S. Angrot.tu, Mosaici antichi in Italia: Sardinia, Rome, 1981, no 114, pl. XLVII (datation suggérée, IV s.). Ce pavement est très proche par les remplissages d'un pavement d'Hippone dont un dessin seulement a été publié : cf. J. Lassus, L'archéologie algérienne en 1957, Libyca, VI, 1958, p. 241, fig. 23.

76 D'autres pavements de maisons en Tunisie et en Algérie, bien datés du IV $\mathrm{V}^{r}$ s., illustrent la composition de cercles en acanthe sécants, sans réunir toutefois les caractéristiques précitées relatives au rendu de l'acanthe et des fuseaux : ainsi à Althiburos, cf. M. ENNAïfer, op. cit. (supra, note 36), p. 129, pl. CLa; ou encore à Cherchel, dans la maison de Thétis et Pélée, cf. J. Lassus, Cherchel. La mosaique de Thétis et Pélée, Bulletin d'Archéologie algérienne, I, 1962-1965, p. 8182 , fig. 4. L'origine africaine de cette variante qu'avait supposée R. Farioli et R. J. A. Wilson (supra, notes 72 et 73), nous semble tout à fait vraisemblable d'après l'ensemble du dossier sur les mosaiques africaines à trames végétalisées de cercles sécants. En revanche, elle paraît discutable à Noël Duval qui croit plutôt à une "inspiration de l'Adriatique» : cf. son compte rendu dans Bulletin monumental, 144, 1986, p. 6466 , en part. note 19 , à propos de l'article de K. Dunbabin, Cahiers des Etudes anciennes, XVII, 1985, p. 8-29; voir aussi sa communication au $\mathrm{V}^{e}$ Colloque international sur la mosaïque antique, Bath 5-12 septembre 1987 (Origine et cheminement du répertoire de la mosaïque byzantine en Occident), Actes sous presse.

77 Parmi les exemples attribuables au ve s., on citera le pavement de la basilique d'Ilemmaberg (cf. S. Lusuardi Siena, supra, note 72) et le pavement du niveau inférieur du bas-côté de S. Maria delle Grazie à Grado dont la datation reste toutefois controversée (L. Bretacchi, Da Aquileia a Venezia, Milan, 1980, p. 295-298). D'autre part, le pavement du baptistère souterrain à Carthage-Sayda pourrait se situer vers la fin du v's. (infra, note 82).

78 Voir la liste donnée par S. Lusuardi Siena (supra, notes 72 et 74 ). La mosaïque du Kef est aussi à rattacher à l'époque byzantine.
Tout d'abord, les mosaïques de Séviac et de Cadeilhan n'ont pas de petit cercle au centre des quatre-feuilles : les cercles sont en effet représentés non contigus, déterminant des petits carrés concaves. Ce principe qui avait été peul-êlre ulilisé dans la basilique d'Oued Ramel en Tunisie ${ }^{79}$ se retrouve sur un pavement tardif de Sousse à décor de cercles sécants, alternativement denticulés et en tiges d'acanthe ${ }^{80}$. Il semble correspondre à un autre mode de tracé des cercles sécants végétalisés, attesté en Byzacène dès l'époque antonine ${ }^{81}$.

La mosaïque de Cadeilhan-Saint-Clar, dans la mesure où elle comporte des filets dentelés à l'intérieur des fuseaux, peut être rapprochée plus étroitement de la série d'Afrique et de l'Adriatique. La nature des ornements de remplissage et en particulier les fleurons à gros cœur circulaire d'où rayonnent alternativement quatre lotus et quatre pétales en fuseau, suggéreraient un parallèle plus précis avec la mosaique du baptistère souterrain à Carthage Sayda (fig. 15) ${ }^{82}$. C'est aussi le même type de fleuron qui revient à Séviac mais la composition ne présente pas le système de quadrillage de filets selon les fuseaux, normalement attesté dans cette variante $^{83}$.

En conclusion, les mosaïstes aquitains ont encore une fois adopté dans leur répertoire des structures végétalisées d'inspiration africaine qui apparaissent typiques de certaines régions de l'Empire à une époque très tardive. Le caractère incertain de nombreuses datations empêche de suggérer une chronologie précise pour les exemples du Sud-Ouest, mais au vu de l'ensemble du dossier, une datation postérieure au $\mathrm{IV}^{\mathrm{s}} \mathrm{s}$. peut être retenue.

79 P. Gaucki.er, Basiliques chrétiennes de Tunisie, Paris, 1913, pl. XVIII.

80 L. Foucher, Inventaire des mosaiques. Sousse, Tunis, 1960, no 57.227, pl. LII-LIII (la datation suggérée, fin du IVe s., parait assurément trop haute).

81 Voir Actes des journées d'étude franco-tunisiennes (supra, note 56).

82 N. Duval, et A. Lézinf, Nécropole chrétienne et baptistère souterrain à Carthage, Cahiers archéologiques, $\mathrm{X}$, 1959 , p. 71-147, en part. p. 90-91, fig. 42-43.

83 La mosaïque du Kef (supra, note 74) ne présente pas de filet axial dans les fuscaux. C'ćtait pcut-ĉtrc aussi le cas du pavement de la basilique d'Oued Ramel connu seulement par un dessin (cf. supra, note 79). 

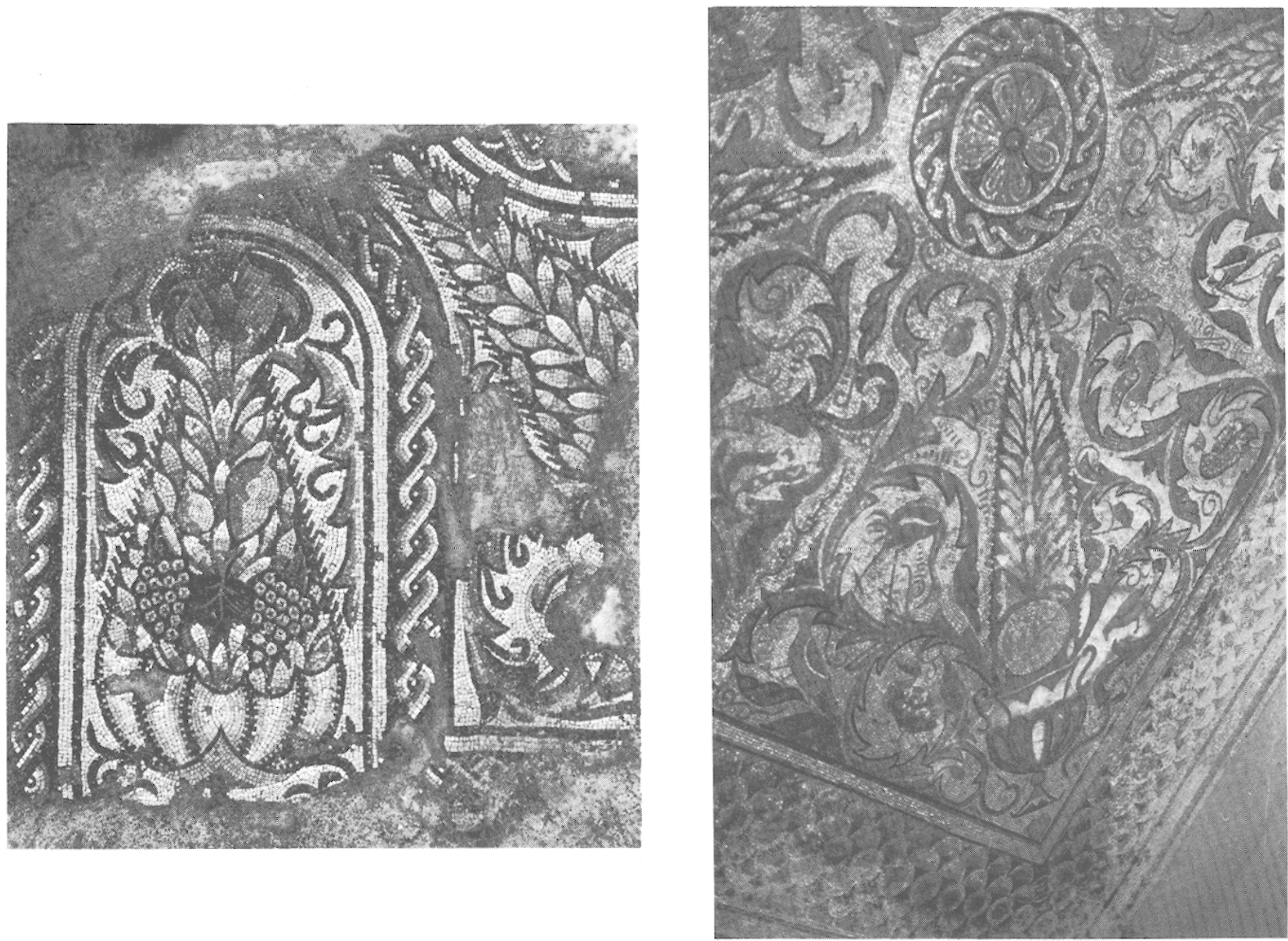

A gauche. Fig. 16 - Séviac. Tapis à pyramides végétales qui décorait l'une des salles d'apparat.

A droite. Fig. 17 - Carthage. Mosaique à pyramides végétales exposée au Musée du Bardo (sol de la salle des Aphrodites de Mahdia).

\section{L'ACANTHE, ORNEMENT ASSOCIE À UNE PYRAMIDE VÉGÉTALE}

La mosaïque de la grande salle d'apparat à abside, située à l'angle n.-e. du péristyle de Séviac, représente des feuilles d'acanthe enserrant deux pyramides végétales dont l'une est placée dans un panneau d'angle selon la diagonale, l'autre dans un panneau en U (fig. 16). Malgré l'aspect fragmentaire du tapis, nous avons pu supposer que les pyramides s'intégraient dans une composition centrée de type cruciforme avec quatre panneaux en $\mathrm{U}^{84}$.

La pyramide de laurier, dans le panneau en $\mathrm{U}$, chargée de fruits (grappes de raisin, cédrat, poire?), montre deux feuilles d'acanthe qui se greffent symétriquement au sommet du cône. Chacune d'entre elles se subdivise en deux tiges à feuilles

84 G. Balmelle, Recueil mos. Gaule, IV, 2, no 292. unilatérales d'orientation opposée : celles du haut enserrent la pointe de la pyramide, en formant un bouquet fermé; celles du bas dessinent une sorte d'aileron. Dans le panneau d'angle très lacunaire, on distingue de part et d'autre du cône de laurier des portions de feuilles d'acanthe qui devaient se développer dc façon symétrique suivant le même principe que dans le panneau précédent : les feuilles du haut encadraient étroitement le cône, celles du bas se recourbaient en une large volute occupant l'espace laissé libre dans le panneau.

L'acanthe rendue de façon très schématique est marquée par des dents aiguës (rouge, jaune, vert), au contour noir et par d'épaisses vrilles rouges et noires. Les nervures sont dessinées en gris bleuté ou en jaune clair.

Ce mode d'utilisation de l'acanthe, exceptionnel en Aquitaine méridionale, suggère des éléments de comparaison avec une série de mosaiques tardives se rattachant à des régions bien définies de l'Empire : 
principalement Proconsulaire et Byzacène ${ }^{85}$, Sicile ${ }^{86}$, Italie du Nord ${ }^{87}$, enfin Péninsule ibérique ${ }^{88}$. Dans le reste de la Gaule, des structures décoratives analogues sont attestées à Saint-Rémy-la-Varenne ${ }^{89}$ et sans doute à Loupian $^{90}$. Comme à Séviac, ces représentations d'acanthe et de pyramides végétales se rencontrent dans des compositions centrées, avec des pyramides placées selon les diagonales ${ }^{91}$. Mais contrairement à ce qui a été observé en Aquitaine, les éléments végétaux organisent eux-mêmes le décor et ne sont pas enfermés à l'intérieur d'un cadre géométrique. L'acanthe prend en général un développement plus important au point de recouvrir, sur de nombreux tapis, tout l'espace compris entre les pyramides $^{92}$. La disposition des feuilles n'est pas

85 Il s'agit de mosaïques de Carthage (deux exemples), de Pupput, de Dougga et de Moknine. Sur les mosaiqques de Carthage et de Pupput, cf. A. Ben Abrd, Une mosaique à pyramides végétales de Pupput, Mosaique. Recueil d'hommages à Henri Stern, Paris, 1983, p. 61-64, pl. XLII, XLVI.1, CCXXX. Sur la mosaique de Moknine, cf. M. YAcoub, A propos d'une mosaique d'époque vandale de Tunisie, Bulletin archéologique du Comilé des Travaux historiques et scientifiques, nouv. sér., fasc. 19B, Paris, 1985, p. 327-340, fig. 1-2. La mosaïque de Dougga est inédite.

86 Mosaiqque de la villa de Tellaro : G. Voza, Aspetti e problemi dei nuovi monumenti d'arte musiva in Sicilia, III Colloquio internazionale sul mosaico antico, Ravenna, 610 settembre 1980, Ravenne, 1984, p. 5-18, en particulier p. 8, fig. 3-4.

87 Mosaïque du Sacellum de S. Severo à Ravenne : cf. R. FARIOLI, op. cit. (supra, note 72), p. 29-30, fig. 12.

88 Mosaique de la villa de Puigvert de Agramunt (province de Lérida): cf. R. Pita Mercé, Diez Coronel, Informe sobre el hallazgo de unos mosaicos romanos en la partida reguer de Puigvert de Agramunt, Noticiario arqueologico hispanico, VI, 1962, p. 171-176, pl. XLVII-XLVIII.

89 G. Dufour, La mosaique de Saint-Rémy (au Musée Turpin de Crissé), dans La province d'Anjou, Angers, 1933, p. 9 (fin Ive/milieu ve s.?).

90 H. Lavagne, R. Prudhomme, D. Rouquette, La villa gallo-romaine des Près-Bas à Loupian (Hérault), Gallia, 34,1976 , p. 230-234, fig. 12, datation suggérée, début du ve $s$. La mosaïque est très fragmentaire mais le relevé semble bien indiquer la représentation d'une pyramide (il n'en subsiste qu'une fraction de bords dentelés).

91 On remarquera toutefois que sur un pavement de Tébessa, la pyramide associée à des rinceaux d'acanthe est placee dans l'axe de l'abside : cf. J.-P. Boucher, Le temple rond de Tébessa Khalia, Libyca, IV, 1956, p. 15, fig. 9. Sur ce monument, voir en dernier lieu, N. Duval, Encore les monuments à auges d'Afrique, Tébessa Khalia, Ir Faraoun, Mélanges de l'École française de Rome, Antiquité, 88, 1976, p. 929-959, part. fig. 9 .

92 Par exemple à Carthage : A. Ben ABEd, op. cit. (supra, note 85), pl. XLV.1; ou encore à Moknine, cf. M. YAcoub, op. cit. (supra, note 85), et Ravenne, cf. R. FARIOLI, op. cit. (supra, note 87). toujours la même; seules quelques mosaïques montrent, comme à Séviac, des enroulements qui viennent se greffer sur la pyramide et qui s'ordonnent symétriquement par rapport à celle-ci : c'est le cas à Tellaro ${ }^{93}$, à Carthage (fig. 17) (94 $^{94}$ et à Ravenne, dans le sacellum de S. Severo ${ }^{95}$.

Ces images de pyramides végétales associées à de l'acanthe correspondent, selon nous, à un répertoire caractéristique à l'origine des ateliers africains et il conviendrait de verser aussi à ce dossier divers pavements de Proconsulaire qui montrent des pyramides liées à d'autres types d'ornements ${ }^{96}$. En Gaule même, il est significatif de constater que tous les exemples de tapis ornés de pyramides sont concentrés dans le Sud-Ouest ${ }^{97}$. Ainsi une fois encore, les mosaïstes aquitains ont repris des éléments spécifiques du répertuire des ateliers africains et les ont en quelque sorte renouvelés, les intégrant dans d'autres compositions ornementales ${ }^{98}$.

Enfin, on ne manquera pas de souligner la présence d'un répertoire analogue sur les sarcophages de l'École d'Aquitaine. Plusieurs d'entre eux montrent en effet un décor d'acanthe associè à une représentation de pyramide végétale : il s'agit soit de rinceaux qui se développent symétriquement à partir d'une pyramide ${ }^{99}$, soit plus rarement, de feuilles d'acanthe enserrant comme à Séviac une pyrami$\mathrm{de}^{100}$. Ces derniers décors se trouvent toujours sur les petits côtés triangulaires du couvercle.

\section{**}

93 G. VozA, op. cit. (supra, note 86); la guirlande de laurier toutefois ne forme pas à proprement parler une pyramide.

94 A. Ben Abed, op. cil. (supra, note 85), pl. XLV.1.

95 R. FARIoLI, op. cit. (supra, note 87 ).

96 On songera en particulier à la mosaiqque de l'oecus de la maison de Nicentius à Thuburbo Majus et à la mosaïque de Carthage provenant de l'aire du "Monument jouxtant la maison des Auriges Grecs" : voir A. BEN ABED, op. cit. (supra, note 85$)$, p. 63 , pl. XLV.2. no 249.

97. C. Balmelle, Recueil mos. Gaule, IV, 1, no $60 ;$ IV, 2 ,

98 Très révélateur à cet égard est le pavement découvert rue Arnaud-Miqueu, à Bordeaux (cité dans Recueil mos. Gaule, IV, 1, no 60, observations).

99 Voir notamment B. Briesenick, op. cil. (supra, note 44), pl. 27.1.

100 Ainsi sur un sarcophage de Moissac : E. LE BLANT, Sarcophages chrétiens de la Gaule, Paris, 1886, no 147, pl. XXXV.1. Même décor sur un sarcophage de Paris: J. B. WARD-Perkins, op. cit. (supra, note 44), pl. XXXVII.2. 
Ces multiples images de décors en acanthe sur les mosaïques de Séviac invitent à s'interroger sur ces ateliers du Sud-Ouest qui, dans l'Antiquité tardive, renouvellent de façon étonnante le répertoire de la mosaïque en Gaule. Les nombreuses similitudes relevées avec les ornements végétaux des mosaïques africaines et plus spécialement de Proconsulaire ${ }^{101}$

101 L'examen détaillé de certaines trames géométriques irait également dans le même sens : cf. C. Barmer.a., A propos d'une mosaïque tardive de Bordeaux, Recueil d'hommages à Henri Stern, Paris, 1983, p. 21-32.

N.B. - Les dessins et relevés sont dus à Marie-Pat Raynaud (fig. 1a, b, c; 2a, b; 3a; 4;8;13b); Mary Duler- nous apparaissent tout à fait signifiantes et laisseraient supposer des rapports privilégiés entre la Gaule du Sud-Ouest et la province d'Afrique, durant l'Antiquité tardive.

\author{
Paulette Aragon-Launet \\ et Catherine Balmelle
}

Larrieu $\dagger$ (fig. 14). Les photographies sont dues à Jean-Pierre Darmon (fig. 12); Didier Dubois (fig. 3b; $5 ; 7 ; 13 a ; 16$ ); W. A. Graham (fig. 11); Jean-Claude Hurteau, Institut de Recherche sur l'Architecture Antique, bureau du Sud-Ouest (fig. 9); Henri Lavagne (fig. 10); Musée du Bardo (fig. 15, 17).

Ma reconnaissance amicale va à Aïcha Ben Abed et à Ouassila Ben Osman qui nous ont communiqué les clichés correspondant aux figures 11 et 17. 